\title{
Effects of musicality and motivational orientation on auditory category learning: A test of a regulatory-fit hypothesis
}

\author{
J. Devin McAuley • Molly J. Henry • Alan Wedd • \\ Timothy J. Pleskac • Joseph Cesario
}

Published online: 18 October 2011

(C) Psychonomic Society, Inc. 2011

\begin{abstract}
Two experiments investigated the effects of musicality and motivational orientation on auditory category learning. In both experiments, participants learned to classify tone stimuli that varied in frequency and duration according to an initially unknown disjunctive rule; feedback involved gaining points for correct responses (a gains reward structure) or losing points for incorrect responses (a losses reward structure). For Experiment 1, participants were told at the start that musicians typically outperform nonmusicians on the task, and then they were asked to identify themselves as either a "musician" or a "nonmusician." For Experiment 2, participants were given either a promotion focus prime (a performance-based opportunity to gain entry into a raffle) or a prevention focus prime (a performance-based criterion that needed to be maintained to avoid losing an entry into a raffle) at the start of the experiment. Consistent with a regulatory-fit hypothesis, self-identified musicians and promotion-primed participants given a gains reward structure made more correct tone classifications and were more likely to discover the optimal disjunctive rule than were musicians and promotionprimed participants experiencing losses. Reward structure (gains vs. losses) had inconsistent effects on the performance of nonmusicians, and a weaker regulatory-fit effect was found for the prevention focus prime. Overall, the findings from this study demonstrate a regulatory-fit effect in the domain of auditory category learning and show that motivational
\end{abstract}

J. D. McAuley $(\bowtie) \cdot$ M. J. Henry · A. Wedd · T. J. Pleskac

J. Cesario

Department of Psychology, Michigan State University,

East Lansing, MI 48824, USA

e-mail: dmcauley@msu.edu

M. J. Henry

Max Planck Institute for Human Cognitive and Brain Sciences, Leipzig, Germany orientation may contribute to musician performance advantages in auditory perception.

Keywords Perception · Individual differences · Music cognition

Musicians have demonstrated a number of performance advantages over nonmusician in auditory perception assessments, which are typically attributed to differences in music ability or formal music training. Some of the reported perceptual differences include enhanced pitch discrimination (Schön, Magne, \& Besson, 2004), better time and/or rhythm discrimination (Jones \& Yee, 1997; McAuley \& Semple, 1999), more automatic encoding of melodic contour and interval structure (Fujioka, Trainor, Ross, Kakigi, \& Pantev, 2004), and more precise expectations about musical structure (Cohen, 2000; Koelsch, Schröger, \& Tervaniemi, 1999). Formal music training has also been shown to be associated with larger cortical volume in primary motor, premotor, and auditory areas, and larger corpus callosum volume (Gaser \& Schlaug, 2003; Hyde, Lerch, Norton, Forgeard, Winner, Evans, \& Schlaug, 2009; Schlaug, Jäncke, Huang, Staiger, \& Steinmetz, 1995; Schlaug, Norton, Overy, \& Winner, 2005). Neuroimaging studies have similarly revealed a number of functional brain differences between musicians and nonmusicians that support a music-training advantage. In particular, musicians recruit prefrontal areas involved in working memory to a greater degree than nonmusicians do during rhythm learning (Chen, Penhune, \& Zatorre, 2008), show decreased motor activation relative to nonmusicians during bimanual tapping (Jäncke, Shah, \& Peters, 2000), show greater connectivity between auditory and motor areas than nonmusicians during beat perception (Grahn \& Rowe, 2009), 
and show more efficient encoding of pitch information than nonmusicians in early stages of auditory processing, including in the brainstem (Musacchia, Sams, Skoe, \& Kraus, 2007; Strait, Kraus, Skoe, \& Ashley, 2009; Wong, Skoe, Russo, Dees, \& Kraus, 2007).

One factor that has rarely been considered in laboratory assessments of the auditory perception skills of musicians and nonmusicians is the role of motivation. It is evident that participants bring different motivations to the laboratory when they participate in behavioral experiments. In this regard, individual differences in motivation are typically treated as a random factor. However, when making comparisons between musician and nonmusician, it seems possible that differences in motivational orientation may make systematic (rather than random) contributions to performance. Along these lines, we have informally observed in the lab that some highly trained musicians appear to approach auditory perception tasks as an opportunity to demonstrate their skill, while others treat the same task as a test that they ought to do well on. Similarly, some nonmusicians appear to approach auditory perception tasks as an opportunity to meet a challenge, while others approach the same task as a test on which they should not perform well. Thus, it is presently not clear how systematic differences in motivation may contribute to auditory perception differences between musicians and nonmusicians. To begin to address this issue, the approach taken in this study was to apply an established theoretical framework in the motivation literature - namely, regulatory focus theory - to the domain of auditory perceptual classification, in order to test hypotheses about how motivational differences between musicians and nonmusicians may interact with task characteristics to alter performance

\section{Regulatory focus theory and the concept of regulatory fit}

Regulatory focus theory distinguishes between two motivational orientations present to varying degrees in all people (Higgins, 1997). People in a promotion focus are motivated to become the person they ideally would like to be (i.e., fulfill their hopes and aspirations), whereas people in a prevention focus are motivated to be the kind of person they feel they ought to be (i.e., fulfill their duties and obligations). Given that both promotion and prevention systems are present in all people, it is possible for situational contingencies to temporarily prime or induce a focus (see, e.g., Forster, Grant, Idson, \& Higgins, 2001; Freitas \& Higgins, 2002; Higgins, Idson, Freitas, Spiegel, \& Molden, 2003).

Two differences between promotion focus and prevention focus systems were of particular relevance for the present study. First, during self-regulation, people in a promotion focus are more concerned with attaining currently unattained goals, whereas people in a prevention focus are more concerned with maintaining currently held states (e.g., Brodscholl, Kober, \& Higgins, 2007; Maddox \& Markman, 2010). In tasks with incentives, framing the task to emphasize attainment versus maintenance has been one common way in which promotion and prevention orientations have been primed. For example, participants can be told "you need to attain X number of points to receive the reward" (promotion prime) or "you need to maintain at least X number of points to avoid losing the reward" (prevention prime). The second difference, which derives from the difference in attainment versus maintenance, is that people in each focus are sensitive to different types of outcome information. A promotion focus activates a mode of processing that focuses the motivational system on the presence or absence of gains in the environment. A prevention focus, conversely, activates a mode of processing that focuses the motivational system on the presence or absence of losses in the environment. Thus, an important idea here is that a promotion focus increases sensitivity to gains and nongains, while a prevention focus increases sensitivity to losses and nonlosses (for reviews, see, e.g., Cesario, Higgins, \& Scholer, 2008; Higgins, 2006).

On this view, in any performance situation both the orientation/regulatory focus of the individual (promotion/ prevention) and the reward structure of the task (e.g., gains/ losses) are operative. Regulatory fit (see Higgins, 2000; Higgins et al., 2003) occurs when task incentives are framed in the manner that is preferred by a person's current orientation - that is, when individuals in a promotion focus are given gains task incentives (a gains reward structure), and individuals in a prevention focus are given losses task incentives (a losses reward structure). Conversely, individuals with a promotion or prevention focus experiencing a losses or a gains reward structure, respectively, experience a state of regulatory nonfit. When outcomes are described in a way that is preferred by a person's regulatory focus (i.e., regulatory fit), the result is enhanced motivational strength and greater valuation of the outcome. Considerable research has supported this prediction across a wide range of domains (e.g., Cesario \& Higgins, 2008; Higgins et al., 2003; Latimer, Rivers, Rench, Katulak, Hicks, Hodorowski, \& Salovey, 2008; Spiegel, Grant-Pillow, \& Higgins, 2004; Werth \& Foerster, 2007; for summaries, see Cesario et al., 2008; Higgins, 2000, 2006). Regulatory fit and nonfit, therefore, have important implications for performance on different types of tasks, a possibility to which we now turn.

\section{Regulatory fit and perceptual category learning}

Regulatory focus theory has recently attracted substantial attention within cognitive science in the area of visual 
category learning (Maddox, Baldwin, \& Markman, 2006; Maddox \& Markman, 2010). The present study extends this research to the auditory domain to begin to examine possible interactions between regulatory focus and musicality. In perceptual category-learning tasks, individuals learn to classify stimuli into two or more categories according to an initially unknown rule. Classification tasks of this sort have, in general, been of interest to researchers because of the ability of such tasks to address questions related to multiple category-learning systems (Ashby \& Maddox, 2005; Erickson \& Kruschke, 1998; Kéri, 2003). One influential model is the competition between verbal and implicit systems (COVIS) model proposed by Ashby and colleagues (Ashby, Alfonso-Reese, Turken, \& Waldron, 1998). The COVIS model distinguishes between an explicit, hypothesis-testing learning system and an implicit, procedural-based learning system. COVIS assumes that performance on perceptual category-learning tasks in which the correct classification rule can be expressed in words (e.g., "Category A consists of short blue lines and long red lines, while Category B consists of short red lines and long blue lines") relies primarily on the explicit hypothesis-testing system, whereas performance on perceptual categorylearning tasks in which the correct classification rule requires the integration of information in a manner that cannot be readily described by the participant (e.g., a category boundary that is the diagonal in a two-dimensional perceptual space) relies on the implicit procedural-based learning system.

Extending work on regulatory focus theory, Maddox and colleagues (Grimm, Markman, Maddox, \& Baldwin, 2008; Maddox et al., 2006; Worthy, Markman, \& Maddox, 2009) provided evidence that regulatory fit increases cognitive flexibility and leads to better performance (relative to regulatory nonfit) on rule-based visual category-learning tasks that require cognitive flexibility (i.e., participants need to explore the space of possible rules to arrive at the correct solution), and worse performance (relative to regulatory nonfit) on information integration tasks in which cognitive flexibility is not beneficial. For the latter task, there is not a rule that can be readily expressed in words, and the exploration of different rule-based strategies will not help participants perform well on the task.

Representative of this line of research, Maddox et al. (2006) randomly assigned participants to either to a promotion focus condition emphasizing the attainment of a desirable state or to a prevention focus condition emphasizing the maintenance of a desirable state. Specifically, in the promotion focus condition, participants were informed that if they exceeded a performance criterion they would gain entry into a drawing for a 1-in-10 chance of winning \$50; in the prevention focus condition, they initially received an entry into the drawing, but they were told that they would lose the entry if they failed to maintain a criterion level of performance. (See Brodscholl et al., 2007, and Maddox et al., 2006, for demonstrations that these conditions induce promotion and prevention foci.) Participants then either gained points for correct responses or lost points for incorrect responses. For a rule-based visual perceptual classification task in which cognitive flexibility was beneficial, individuals with a regulatory fit learned the rule more quickly than (and outperformed) individuals with a regulatory nonfit. Conversely, for an information integration task in which cognitive flexibility was a disadvantage, participants with a regulatory nonfit learned the rule more quickly than (and outperformed) individuals with a regulatory fit. Converging evidence for the observed regulatory fit/nonfit effects was found by fitting a number of decision bound models (cf. Ashby \& Maddox, 1993; Maddox et al., 2006) to the data on a block-by-block basis. Notably, the proportion of participants who were best fit by the optimal classification rule when cognitive flexibility was beneficial was larger in regulatory-fit conditions than in regulatory-nonfit conditions; conversely, participants in a regulatory fit were slower to converge to the optimal classification rule for information integration tasks in which cognitive flexibility was a disadvantage.

The present extension of regulatory focus theory to the auditory domain to examine effects of musicality was modeled after Grimm, Markman, Maddox, and Baldwin (2009, Exp.2). Grimm et al. demonstrated that different motivational orientations (i.e., regulatory foci) could be primed in male and female participants by emphasizing gender differences on a visual category-learning task. Specifically, participants were told that the test was diagnostic of gender differences in spatial abilities. It was then explained to participants prior to testing that the test that they were about to take was one on which (1)women typically outperform men, or on which (2)men typically outperform women. Then, participants performed the category-learning task while either gaining points for correct responses (a gains condition) or losing points for incorrect responses (a losses condition). The visual stimuli were lines that varied on three dimensions (length, orientation, and position), and optimal performance was achievable by learning to classify stimuli according to a two-dimensional conjunctive rule. Grimm et al. hypothesized that men and women given positive stereotypes would focus on goal attainment and adopt a promotion focus, while men and women given negative stereotypes would focus on avoiding poor performance and adopt a prevention focus. Consistent with a regulatory-fit interpretation, the authors found that women given a positive stereotype outperformed men given a negative stereotype for the gains condition, while the effect was reversed when the primed stereotypes were reversed for men and women. 


\section{Present study}

Here, we applied the approach of Grimm et al. (2009) to the auditory domain in order to investigate the contributions of motivational orientation to musician/nonmusician differences in auditory skills. Participants were first told that they would take a listening test that musicians typically perform better on than nonmusicians and were then immediately asked to identify themselves as a musician or a nonmusician. The type of test we chose to examine was an auditory categorylearning task, which required participants to learn to correctly classify tone stimuli that varied along two acoustic dimensions (frequency and duration). Optimal performance on the task required learning a verbalizable exclusive disjunctive rule that required cognitive flexibility and for which regulatory fit was hypothesized to be advantageous (see Fig. 1). Tones short in duration and low in pitch or long in duration and high in pitch belonged to Category A, while tones short in duration and high in pitch or long in duration and low in pitch belonged to Category B.

With respect to the effect of musicality, we hypothesized that priming self-identified musicians with a positive stereotype and then giving them a novel and challenging task would lead musicians to focus on goal attainment and adopt a promotion focus. From a regulatory fit perspective, this means that musicians should perform better in the gains condition (a regulatory fit) than in the losses condition (a regulatory nonfit). In contrast, for nonmusicians, it seemed possible that the prime ("musicians typically perform better on the test than nonmusicians) might have weak or null effects. Notably, previous research on stereotype threat has shown that the effectiveness of priming a negative stereotype depends on the importance that individuals place on the ability in question (e.g., women primed with a negative stereotype about math performance were more affected by the prime if they assigned high importance to mathematical ability; Cadinu, Maass, Frigerio, Impagliazzo, \& Latinotti, 2003). Thus, extending this idea to a consideration of music expertise suggests that if selfidentified nonmusicians assign less importance to musical ability than do musicians, they might also be less likely to be influenced by the performance prime. This implies that for the comparison between musicians and nonmusicians, the effects of the prime on performance differences between groups should be driven primarily by the musicians. Moreover, from a regulatory-fit perspective, group differences, if present, should be larger in the gains condition (a regulatory fit for the musicians) than in the losses condition (a regulatory nonfit for the musicians).

A second experiment was conducted (1)to more directly examine the role of regulatory fit in auditory perceptual classification and (2)to provide converging evidence for the hypothesis that musicians adopted a promotion focus in Experiment 1. Experiment 2 replicated Experiment 1, but rather than comparing musicians and nonmusicians, we explicitly primed promotion and prevention foci using a raffle ticket manipulation (Maddox et al. 2006). Participants with a regulatory fit (promotion-gains, prevention-losses) were predicted to achieve higher levels of classification accuracy and to learn the disjunctive classification rule better than participants with a regulatory nonfit (promotion-losses, prevention-gains).
Fig. 1 Distributions of Category $\mathrm{A}$ and Category B stimuli. Tones low in pitch and short in duration or high in pitch and long in duration were assigned to Category A (black circles), whereas tones low in pitch and long in duration or high in pitch and short in duration were assigned to Category B (white circles), forming an exclusive disjunctive classification rule. Tone duration ranged between 100 and $900 \mathrm{~ms}$, and tone frequency was varied in logfrequency units using a semitone scale over an octave range between E4 $(329 \mathrm{~Hz})$ and $\mathrm{E} 5$ $(658 \mathrm{~Hz})$. The optimal decision bound for the exclusive disjunctive rule is shown by the dotted lines

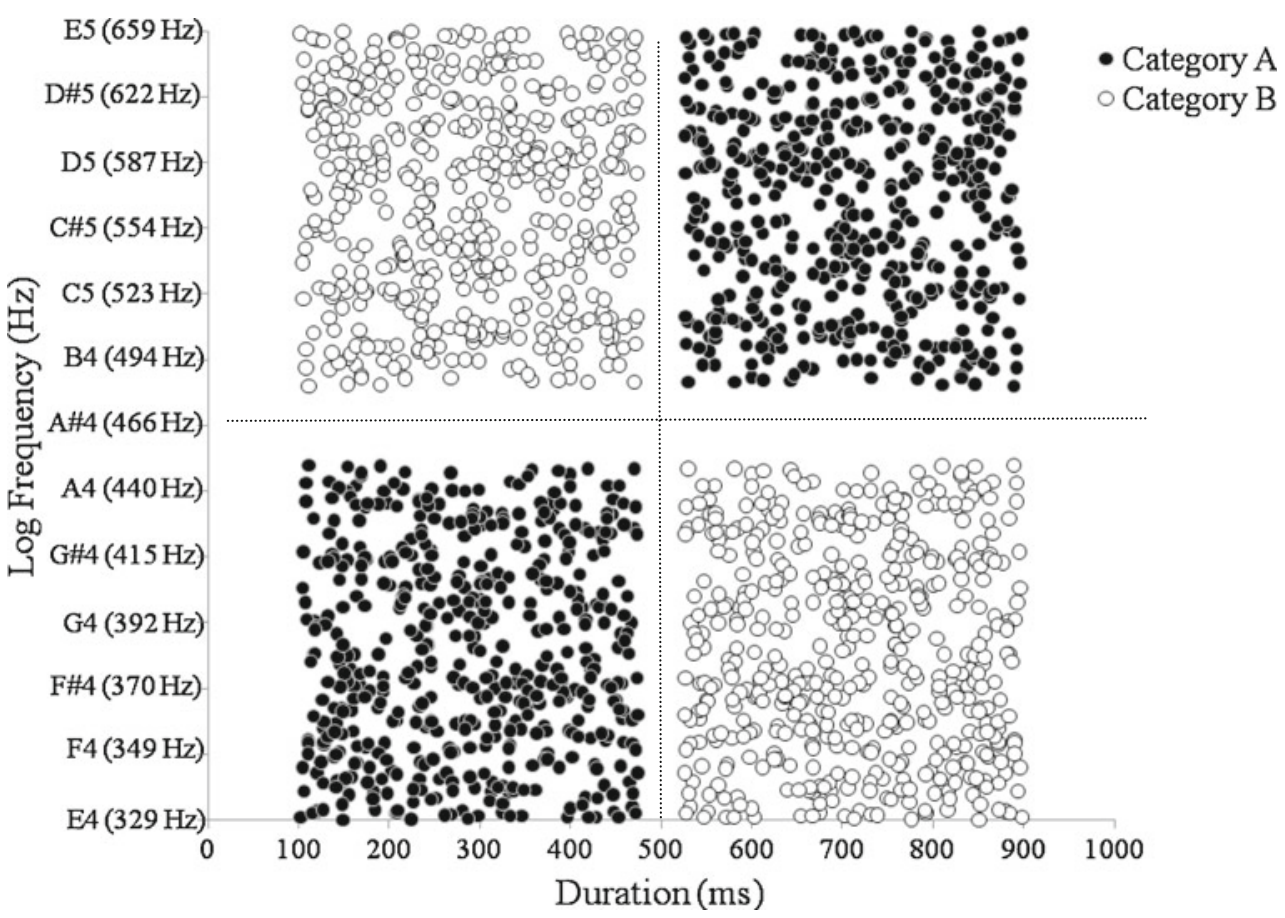




\section{Experiment 1}

\section{Method}

\section{Design and participants}

The experiment had a 2 (musicality: self-identified musicians vs. self-identified nonmusicians) $\times 2$ (reward structure: gains vs. losses) $\times 8$ (block) mixed factorial design. Musicality and reward structure were between-subjects factors, while block was a within-subjects factor. A group of 56 individuals from a large Midwestern university community participated in return for extra credit in a psychology course or a cash payment. Participants were randomly assigned to either the gains condition, in which they gained points for correct responses, or a losses condition, in which they lost points for incorrect responses. After being told that the task was diagnostic of their music ability, participants were then asked to self-identify as musicians or nonmusicians. Overall, fewer participants selfidentified as musicians $(n=17)$ than as nonmusicians $(n=$ 39). This yielded four between-subjects conditions (musicians-gains, $n=8$; musicians-losses, $n=9$; nonmusiciansgains, $n=21$; nonmusicians-losses, $n=18$ ).

\section{Stimuli and equipment}

Two-thousand single sine tones were generated that varied along two stimulus dimensions: frequency and duration. The stimuli were sampled from uniform distributions that formed an exclusive disjunctive classification rule; half of the tones were Category A stimuli, and half were Category B stimuli. Figure 1 shows a scatterplot of the stimuli along with solid lines marking the optimal disjunctive rule. Frequency varied on a log-frequency scale over an octave range between $329 \mathrm{~Hz}$ (E4) and $658 \mathrm{~Hz}$ (E5). The frequency value that maximally separated stimulus categories was $466 \mathrm{~Hz}$. Duration varied in milliseconds over an 800 -ms range between 100 and $900 \mathrm{~ms}$. The duration value that maximally separated stimulus categories was $500 \mathrm{~ms}$. Category A stimuli consisted of either short-duration lowfrequency tones $(100-475 \mathrm{~ms}, 329-450 \mathrm{~Hz})$ or longduration high-frequency tones (525-900 ms, 482$658 \mathrm{~Hz}$ ). Category B stimuli consisted of either shortduration high-frequency tones $(100-475 \mathrm{~ms}, 482-658 \mathrm{~Hz})$ or long-duration low-frequency tones $(525-900 \mathrm{~ms}, 329$ $450 \mathrm{~Hz}$ ). The stimuli were generated offline using Praat software (Boersma \& Weenink, 2005) and presented during the experiment at a comfortable listening level over Sennheiser HD-280 Pro headphones (Old Lyme, CT); stimulus presentation and response collection were controlled by E-Prime software (Psychology Software Tools, Inc.) running on a Dell PC computer.

\section{Procedure}

Participants were first administered a number of surveys assessing whether the self-identified musician and nonmusician groups were a priori different with respect to a number of self-report measures, including "motivation to do well on the task." Following Grimm et al. (2009), all participants initially completed the Regulatory Focus Questionnaire (RFQ: Higgins et al., 2001), the Beck Anxiety Inventory (BAI: Beck, Epstein, Brown, \& Steer, 1988), and the Penn State Worry Questionnaire (PSWQ: Meyer, Miller, Metzger, \& Borkovec, 1990). The RFQ was used to assess potential differences between musicians and nonmusicians in chronic regulatory focus. It assesses an individual's history of promotion success and prevention success by asking them to rate how often certain events have happened in their past (e.g., "How often did you obey rules and regulations that were established by your parents," "Not being careful enough has gotten me into trouble at times"). The BAI and PSWQ were administered because they measure two constructs, anxiety and worry, respectively, which have the potential to be related to a chronic prevention focus. The BAI asks participants to indicate how much they had been bothered by a variety of symptoms in the last week (e.g., "nervous," "faint," "terrified"). Cronbach's alpha coefficient for the BAI was .84. The PSWQ asks participants to rate how typical of them they consider statements about worrying (e.g., "My worries overwhelm me," "When I am under pressure I worry a lot."). Responses on the BAI range from $0=$ Not at all to $3=$ Severely, I could barely stand it. Responses on the PSWQ range from $1=$ Not at all typical of me to $5=$ Very typical of me. Cronbach's alpha coefficient for the PSWQ was .93.

Participants were next told the following: "This is an experiment testing musical training differences in listening abilities. Previous research has shown that musicians perform better than nonmusicians on tests of listening ability." Participants were then asked to identify themselves as either a musician or a nonmusician by pressing the "M" or " $\mathrm{N}$ " key on the computer keyboard, respectively. Next, participants were asked to provide ratings in response to the following questions: "How well do you think you will perform on this test" $(1=$ very badly, $9=$ very well $)$, "How well do you think you will like the test" $(1=$ not at all, $9=$ very much), and "How motivated are you to do well on the test" $(1=$ not at all, $9=$ very motivated $)$. Participants then completed the Positive Affect Negative Affect Schedule (PANAS: Watson, Clark, \& Tellegen, 1988), which is a 20adjective checklist that asks participants to rate the degree to which each adjective on the list describes their current emotional state. Responses range from $1=$ Very slightly or not at all to $5=$ Extremely. The PANAS yields a positive affect (PA) scores and a negative affect (NA) score. An 
example of a PA item is "enthusiastic," while an example of an NA item is "irritated."

During testing, participants were presented with a single tone on each trial that was randomly sampled from the stimulus space shown in Fig. 1, and they indicated whether the tone was from Category A or B by pressing one of two labeled response box buttons. Throughout the experiment, a point meter was displayed on the right side of the screen that tracked the number of points gained or lost, and additionally displayed the to-be-achieved or to-be-avoided criterion score. Participants given the gains reward structure gained 2 points for each correct response and 0 points for each incorrect response, with a to-be-achieved criterion score of +58 points (at least $80.5 \%$ correct). For correct responses, the computer screen displayed " +2 ," the point meter increased to show participants that they were closer to the to-be-achieved criterion score, and participants heard a cash register ("ka-ching") sound to increase the saliency of the point reward. For incorrect responses, the computer screen displayed " +0 " and the point meter did not change. Participants given the losses reward structure lost 3 points for each incorrect response and 1 point for each correct response, with a to-be-avoided criterion score of -58 points (no worse than $80.5 \%$ correct). For incorrect responses, the computer screen displayed " -3 ," the point meter decreased to show participants that they were closer to the to-beavoided criterion score, and participants heard a "buzzer" sound to increase the saliency of the point loss. For correct responses, the computer screen displayed " -1 " and the point meter decreased by a smaller amount than when losing 3 points. Once a participant had completed a trial block, he or she was given feedback about the status of the performance on the task; participants were told whether or not they had successfully achieved the criterion score (gains condition) or avoided the criterion score (losses condition) and were reminded that for this test musicians were generally successful in achieving or avoiding the criterion score, respectively. In total, there were eight blocks of trials, with 36 trials per block; participants did not know how many blocks they would complete.

Immediately after completing the perceptual classification task, participants completed the PANAS for a second time; for the PANAS, the alpha coefficients for the PA measure were .83 and .86 for the pre- and posttest assessments, respectively, while those for the NA measure were .79 and .78 for the pre- and posttest assessments, respectively. Participants also responded to a series of music-related statements on a scale ranging from $1=$ strongly disagree to $9=$ strongly agree. These appeared in the following order: "I am good at music," "It is important to me that I am good at music," "My musical ability is important to my identity." Participants were next asked to make ratings in response to the following questions, on a scale ranging from $1=$ very badly to $9=$ very well: "How well do you believe you performed overall on the test," "How well do you think you performed compared to musicians," and "How well do you think you performed compared to nonmusicians." Finally, participants completed a posttest questionnaire that assessed their ratings of natural musical ability $(1=$ very poor, $6=$ very good $)$, level of effort $(1=I$ did not try at all, $6=$ I tried my best $)$, level of attention $(1=I$ did not pay attention, $6=$ I paid full attention $)$, level of task difficulty $(1=$ not difficult at all, $6=$ very difficult $)$, and level of task understanding $(1=I$ did not understand at all, $6=I$ understood exactly what to do). The experiment lasted approximately $90 \mathrm{~min}$.

\section{Results}

\section{Comparison of self-identified musician and nonmusician samples on self-report measures}

Tables 1 and 2 report means and standard deviations for all pre- and posttest items and scores for the different survey measures for the sample of self-identified musicians $(n=$ $17)$ and the sample of self-identified nonmusicians $(n=39)$. As expected, the self-identified musicians and nonmusicians differed in their responses to a number of self-report items concerning musical ability and general interest in music. Musicians reported receiving more years of formal musical training $(M=7.5$ years, $S D=2.8)$ than nonmusicians $(M=1.1$ years, $S D=2.1), t(54)=9.53, p<.001$. Ratings for the statement "I am good at music" were significantly higher for musicians $(7.0 \pm 1.9)$ than for nonmusicians $(3.4 \pm 1.9), t(54)=6.61, p<.001$. Musicians also judged that it was more "important to be good at music" than did nonmusicians $(6.7 \pm 1.9$ vs. $3.2 \pm 2.0)$, $t(54)=5.99, p<.001$; provided higher ratings to "musical ability is important to my identity" $(6.0 \pm 1.8$ vs. $2.5 \pm$ $1.7), t(54)=6.76, p<.001$; and rated their natural musical ability as higher than that of nonmusicians $(4.5 \pm 1.2 \mathrm{vs}$. $2.5 \pm 1.0), t(54)=5.83, p<.001$.

Next, the self-identified musician and nonmusician samples were compared in their responses to the pretest questions. Just after being told that musicians tended to perform better than nonmusicians on the test that they were about to take, self-identified musicians judged that they would perform better than the nonmusicians (musicians, $M=6.3, S D=1.4$; nonmusicians, $M=5.1, S D=1.7), t(54)=2.64, p=.01$; felt than they would like the test more than did the nonmusicians (musicians, $6.6 \pm 1.7$; nonmusicians, $5.2 \pm 1.5$ ), $t(54)=3.13$, $p=.01$; and were slightly more motivated than the nonmusicians to do well on the test (musicians, $M=7.5 \pm$ 1.4; nonmusicians, $M=6.6 \pm 1.8), t(54)=1.75, p=.09$. Musicians and nonmusicians did not differ in chronic promotion focus, chronic prevention focus, PSWQ scores, 
Table 1 Mean ratings $( \pm S D)$ for self-report items for musicians and nonmusicians
Significant differences: ${ }^{*} p<.05$, ${ }^{* *} p<.01$.

\begin{tabular}{lll}
\hline Pretest Items & Musicians & Nonmusicians \\
\hline How well do you think you will perform on this test? & $6.3(1.4)^{* *}$ & $5.1(1.7)$ \\
How well do you think you will like the test? & $6.6(1.7)^{* *}$ & $5.2(1.5)$ \\
How motivated are you to do well on the test? & $7.5(1.4)$ & $6.6(1.8)$ \\
Posttest Items & & \\
Formal music training (years) & $7.5(2.8)^{* *}$ & $1.1(2.1)$ \\
I am good at music. & $7.0(1.9)^{* *}$ & $3.4(1.9)$ \\
It is important for me to be good at music. & $6.7(1.9)^{* *}$ & $3.2(2.0)$ \\
My musical ability is important to my identity. & $6.0(1.8)^{* *}$ & $2.5(1.7)$ \\
How well did you perform overall? & $3.7(2.4)$ & $4.4(2.3)$ \\
How well did you perform compared to musicians? & $4.3(2.5)$ & $3.1(2.2)$ \\
How will did you perform compared to nonmusicians? & $5.7(2.3)$ & $5.5(2.2)$ \\
Natural musical ability & $4.5(1.2)^{* *}$ & $2.5(1.0)$ \\
Level of effort & $5.3(0.9)$ & $4.8(1.2)$ \\
Level of attention & $4.7(1.2)$ & $4.5(1.1)$ \\
Level of task difficulty & $4.6(1.2)$ & $4.5(1.3)$ \\
Level of task understanding & $3.9(1.7)$ & $3.7(1.5)$ \\
\hline
\end{tabular}

BAI scores, or PA and NA scores on the PANAS assessed either before or after completion of the tone classification task (all $p \mathrm{~s}>.2$ ).

The musician and nonmusician samples also did not differ in their posttest ratings of effort expended, attention to the task, task difficulty, or task understanding (all $p \mathrm{~s}>$ .13) or in their self-assessments of how they performed on the task relative to musicians and nonmusicians (all $p \mathrm{~s}>$ .1). Additional comparisons on posttest items showed no effects of reward structure and no interactions between musicianship and reward structure (all $p \mathrm{~s}>.2$ ).

\section{Perceptual classification performance}

Accuracy analyses Figure 2 shows proportions of correct tone classifications (PC) as a function of trial block for selfidentified musicians (solid lines) and nonmusicians (dotted lines), given a gains reward structure (filled markers) and a

Table 2 Mean scores $( \pm S D)$ for each survey measure for the samples of self-identified musicians and nonmusicians

\begin{tabular}{lll}
\hline Survey Measure & Musicians & Nonmusicians \\
\hline RFQ (promotion) & $18.8(2.1)$ & $19.6(2.3)$ \\
RFQ (prevention) & $15.2(2.5)$ & $14.9(2.9)$ \\
BAI & $9.7(6.6)$ & $8.2(6.4)$ \\
PSWQ & $48.1(18.5)$ & $47.3(13.5)$ \\
PANAS PA (pre) & $32.2(8.2)$ & $29.8(6.8)$ \\
PANAS NA (pre) & $13.2(2.9)$ & $13.4(4.3)$ \\
PANAS PA (post) & $19.8(7.2)$ & $20.6(8.1)$ \\
PANAS NA (post) & $17.6(5.4)$ & $16.5(5.3)$ \\
\hline
\end{tabular}

See text for test abbreviations. pre, pretest; post, posttest losses reward structure (open markers). In line with the hypothesis that musicians primed with a positive stereotype would adopt a promotion focus and experience regulatory fit in the gains condition, musicians in the gains condition showed a tendency to outperform all other groups. To assess the reliability of this trend, PC values were initially subjected to a 2 (musicality) $\times 2$ (reward structure) $\times 8$ (block) ANOVA, with participants' responses to the three statements on the pretest ("How well do you think you will perform on the test?", "How much do you think you will like the test?", "How motivated are you to perform well on the test?") that differed between musicians and nonmusicians included as

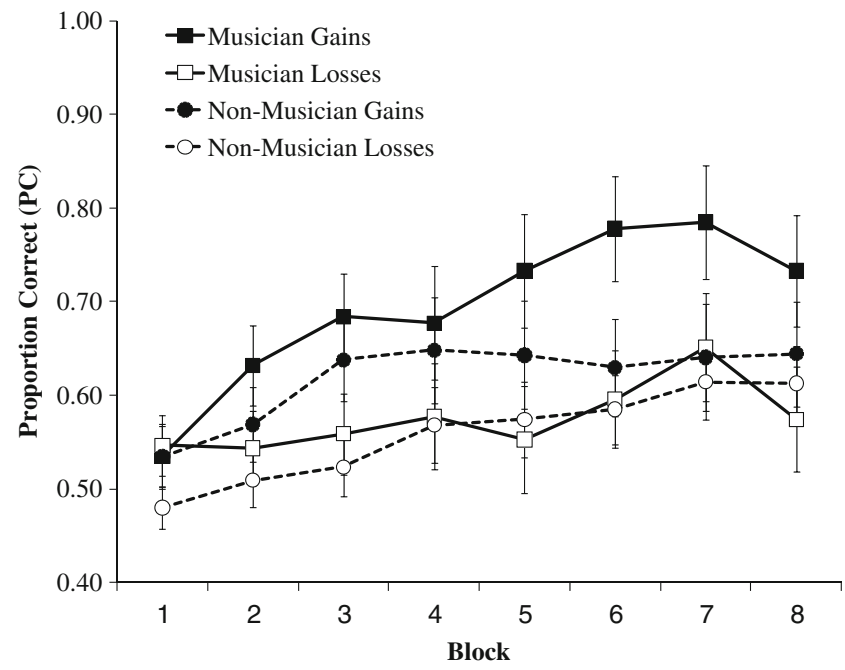

Fig. 2 Proportions of correct classification responses (PC) in Experiment 1 in each block for self-identified musicians (solid lines) and nonmusicians (dotted lines), given the gains reward structure (filled markers) or the losses reward structure (open markers) 
covariates. The ANOVA on PCs revealed a main effect of reward structure, $F(1,49)=7.5, p<.01$, and a marginally significant three-way interaction between musicality, reward structure, and block, $F(7,343)=1.93, p=.06$. There were also interactions between participants' pretest ratings of motivation and block, $F(7,343)=2.18, p<.05$, and their pretest ratings of how much they thought they would like the test and block, $F(7,343)=2.17, p<.05$. There were no other main effects or interactions (all $p \mathrm{~s}>.2$ ). An ANOVA on $d^{\prime}$ rather than PC revealed a similar pattern of results, including a significant three-way interaction between musicality, reward structure, and block, $F(7,343)=2.25, p=.03$.

To unpack the three-way interaction between musicality, reward structure, and block, PC values were regressed on block (1-8) for each participant in order to obtain a slope estimate (rate of change in PCs across block) for each participant. For this analysis, large values for the estimated slope indicated greater improvement in performance over blocks. Of primary interest was a comparison of slopes across conditions. The estimated slopes were subjected to a 2 (musicality) $\times 2$ (reward structure) ANOVA, with participants' responses to the three statements on the pretest ("How well do you think you will perform on the test?", "How much do you think you will like the test?", "How motivated are you to perform well on the test?") included as covariates. There was no main effect of musicality, $F(1,48)=0.22, p=.64$, and no main effect of reward structure, $F(1,48)=0.36, p=.55$, but a two-way interaction between musicality and reward structure, $F(1,48)=4.67, p=.036$, was in the hypothesized direction. Consistent with the hypothesis that musicians primed with a positive stereotype would adopt a promotion focus and experience regulatory fit with a gains reward structure, musicians showed greater improvement over blocks (i.e., a larger slope) in the gains condition $(M=.03$, $S D=.024)$ than in the losses condition $(M=.010, S D=.016)$, $t(11)=2.00, p=.03$, one-tailed. Furthermore, singlesample $t$-tests revealed that the estimated slope for musicians in the gains condition was significantly greater than zero, $t(7)=3.42, p=.01$, whereas the slope for musicians in the losses condition was not different from zero, $t(8)=1.79, p=.11$. Nonmusicians, in contrast, did not show any difference in their degree of improvement over blocks in the two reward structure conditions (gains, $M=$ $.013, S D=.027$; losses, $M=.02, S D=.027), t(33)=-0.69$, $p=.50$ ); the direction of the observed slope difference was opposite to that observed for musicians, and thus consistent with a prevention focus rather than a promotion focus.

Model-based analyses To supplement the accuracy analyses and to examine what participants were learning when making tone classifications, we fit a number of different decision bound models (DBMs) to the choice data at the individualparticipant level on a block-by-block basis. DBMs describe how participants perceive a stimulus in multidimensional space and how they ultimately make a categorization decision based on where that perception falls in the perceptual space. For the present data set, three classes of models were of interest: (a) a class of unidimensional models, (b) a class of disjunctive models, and (c) a random-responder model (see Table 3). The unidimensional models assume that participants make a categorization decision on the basis of one dimension only: frequency or duration. For each stimulus dimension, we fit two unidimensional models. One version assumed that participants would respond with Category A if the perceived stimulus's first dimension value were less than the criterion, or otherwise they would respond with Category

Table 3 Descriptions of the decision bound models that were fit to the data

\begin{tabular}{|c|c|c|}
\hline Model & Description & Parameters \\
\hline Unidimensional Duration 1 & $\begin{array}{l}\text { Participants responded with Category } \mathrm{A} \text { if the duration of the stimulus } \\
\text { was below criterion } \lambda_{\mathrm{d}}\end{array}$ & $\begin{array}{l}\text { Noise parameter } \sigma \text {, Decision criterion } \\
\text { on duration dimension } \lambda_{\mathrm{d}}\end{array}$ \\
\hline Unidimensional Duration 2 & $\begin{array}{l}\text { Participants responded with Category B if the duration of the stimulus } \\
\text { was below criterion } \lambda_{\mathrm{d}}\end{array}$ & $\begin{array}{l}\text { Noise parameter } \sigma \text {, Decision criterion } \\
\text { on duration dimension } \lambda_{\mathrm{d}}\end{array}$ \\
\hline Unidimensional Frequency 1 & $\begin{array}{l}\text { Participants responded with Category A if the frequency of the stimulus } \\
\text { was below criterion } \lambda_{\mathrm{f}}\end{array}$ & $\begin{array}{l}\text { Noise parameter } \sigma \text {, Decision criterion } \\
\text { on frequency dimension } \lambda_{\mathrm{f}}\end{array}$ \\
\hline Unidimensional Frequency 2 & $\begin{array}{l}\text { Participants responded with Category B if the frequency of the stimulus } \\
\text { was below criterion } \lambda_{\mathrm{f}}\end{array}$ & $\begin{array}{l}\text { Noise parameter } \sigma \text {, Decision criterion } \\
\text { on frequency dimension } \lambda_{\mathrm{f}}\end{array}$ \\
\hline Disjunctive: Suboptimal & $\begin{array}{l}\text { Participants separately determined the value of the duration relative to } \\
\text { criterion } \lambda_{\mathrm{d}} \text { and the value of the frequency relative to criterion } \lambda_{\mathrm{f}} \text {, then } \\
\text { combined the representations before choosing a response. Participants } \\
\text { set the location of the criteria. }\end{array}$ & $\begin{array}{l}\text { Noise parameter } \sigma \text {, Decision criterion } \\
\text { on duration dimension } \lambda_{\mathrm{d}} \text {, Decision } \\
\text { criterion on frequency dimension } \lambda_{\mathrm{f}}\end{array}$ \\
\hline Disjunctive: Optimal & $\begin{array}{l}\text { Participants separately determined the value of the duration relative to } \\
\text { criterion } \lambda_{\mathrm{d}} \text { and the value of the frequency relative to the criterion } \lambda_{\mathrm{f}} \text {, } \\
\text { then combined the representations before choosing a response. } \\
\text { Participants used the optimal criteria that maximized proportion correct. }\end{array}$ & Noise parameter $\sigma$ \\
\hline Random Responder & $\begin{array}{l}\text { Participants responded with Category A with probability } p \text {; otherwise, } \\
\text { they responded with Category B. }\end{array}$ & $\begin{array}{l}\text { Probability of responding with } \\
\text { Category A }\end{array}$ \\
\hline
\end{tabular}


$\mathrm{B}$; the other version assumed the opposite mapping. For the class of disjunctive models, we fit an optimal model, in which the two decision criteria were set at the optimal locations used to assign category labels in the experiment, and a suboptimal model that allowed the two decision criteria parameters to vary. Note that the term optimal does not imply that if a participant used the optimal disjunctive rule he or she would obtain $100 \%$ accuracy. The model is stochastic, so optimality implies that the participant would have the highest level of accuracy obtainable given his or her level of perceptual and/or criteria variability. Finally, we considered a random-responder model in which the probability that a participant would indicate Category A or B was set equal to the observed relative frequency of choosing that category.

Of central interest was whether participants would learn to classify the tones according to the optimal disjunctive rule, and how quickly they would do so. The regulatory-fit hypothesis predicted that the use of a disjunctive rule should be discovered and applied more quickly for individuals in a regulatory fit as compared to those in a regulatory nonfit (Maddox et al., 2006). Therefore, if musicians did experience regulatory fit with a gains reward structure, we expected that the percentage of participants using the optimal DBM would be higher for musicians in the gains condition than in the losses condition. To assess this hypothesis, the seven DBMs were fit to the choice data for each participant using the Bayes information criterion
(BIC) as the goodness-of-fit measure (Kass \& Raftery, 1995; Raftery, 1995; Wasserman, 2000). Model fits were performed on the choice data (1)on a block-by-block basis, to examine changes in the decision rule across blocks, and (2)collapsed across blocks, to consider the best-fitting model overall. For the model fits, a 1:1 mapping between the physical stimulus in multidimensional frequencyduration space and perceptual space was assumed, but we allowed for trial-by-trial (unbiased) variability in the percept. The smaller the value of the BIC, the better the fit of the model, regardless of the number of free parameters; see the Appendix for additional modeling details. To further quantify how quickly each participant learned the optimal disjunctive rule, we identified for each participant the total number of blocks and the first block for which the optimal disjunctive model best fit the data; for the first-block measure, participants whose data were never fit best by the optimal disjunctive model were coded as a 9 .

The models fit the data well, explaining $83.5 \%$ of variance. Table 4 summarizes the percentages of participants in each block best fit by the optimal disjunctive model, the suboptimal disjunctive model, the unidimensional frequency model, the unidimensional duration model, and the random-responder model. To facilitate comparisons with the accuracy data reported in Fig. 2, Fig. 3 shows the percentages of participants best fit by the optimal disjunctive model for each block for musicians (gains vs. losses) and nonmusicians (gains vs. losses).
Table 4 Percentages of selfidentified musician and nonmusician participants in the gains and losses incentive conditions in Experiment 1 for which an optimal, a suboptimal, a unidimensional (UD) frequency, a unidimensional duration, or a random-responder model best fit the accuracy (as percentages correct) data according to the BIC metric

\begin{tabular}{|c|c|c|c|c|c|c|c|c|c|}
\hline \multirow[b]{2}{*}{ Model } & \multirow[b]{2}{*}{ Condition } & \multicolumn{8}{|c|}{ Block } \\
\hline & & 1 & 2 & 3 & 4 & 5 & 6 & 7 & 8 \\
\hline \multirow[t]{4}{*}{ Optimal } & Musician gains & 62.5 & 71.4 & 50.0 & 50.0 & 87.5 & 100.0 & 50.0 & 62.5 \\
\hline & Musician losses & 33.3 & 44.4 & 33.3 & 44.4 & 11.1 & 55.6 & 22.2 & 33.3 \\
\hline & Nonmusician gains & 33.3 & 38.1 & 57.1 & 42.9 & 42.9 & 42.9 & 52.4 & 33.3 \\
\hline & Nonmusician losses & 11.1 & 5.6 & 33.3 & 27.8 & 44.4 & 50.0 & 27.8 & 11.1 \\
\hline \multirow[t]{4}{*}{ Suboptimal } & Musician gains & 12.5 & 14.3 & 12.5 & 25.0 & 0.0 & 0.0 & 25.0 & 12.5 \\
\hline & Musician losses & 11.1 & 22.2 & 0.0 & 0.0 & 22.2 & 0.0 & 11.1 & 11.1 \\
\hline & Nonmusician gains & 28.6 & 28.6 & 9.5 & 19.0 & 28.6 & 23.8 & 14.3 & 28.6 \\
\hline & Nonmusician losses & 22.2 & 22.2 & 16.7 & 11.1 & 11.1 & 11.1 & 22.2 & 22.2 \\
\hline \multirow[t]{4}{*}{ UD Freq } & Musician gains & 0.0 & 0.0 & 0.0 & 0.0 & 0.0 & 0.0 & 0.0 & 0.0 \\
\hline & Musician losses & 11.1 & 0.0 & 11.1 & 0.0 & 0.0 & 0.0 & 0.0 & 11.1 \\
\hline & Nonmusician gains & 0.0 & 0.0 & 4.8 & 0.0 & 0.0 & 4.8 & 0.0 & 0.0 \\
\hline & Nonmusician losses & 5.6 & 0.0 & 0.0 & 0.0 & 5.6 & 0.0 & 0.0 & 5.6 \\
\hline \multirow[t]{4}{*}{ UD Dur } & Musician gains & 0.0 & 0.0 & 12.5 & 0.0 & 0.0 & 0.0 & 0.0 & 0.0 \\
\hline & Musician losses & 0.0 & 11.1 & 0.0 & 0.0 & 0.0 & 0.0 & 0.0 & 0.0 \\
\hline & Nonmusician gains & 0.0 & 4.8 & 0.0 & 0.0 & 0.0 & 4.8 & 0.0 & 0.0 \\
\hline & Nonmusician losses & 5.6 & 5.6 & 0.0 & 0.0 & 5.6 & 11.1 & 5.6 & 5.6 \\
\hline \multirow[t]{4}{*}{ Random } & Musician gains & 25.0 & 14.3 & 25.0 & 25.0 & 12.5 & 0.0 & 25.0 & 25.0 \\
\hline & Musician losses & 44.4 & 22.2 & 55.6 & 55.6 & 66.7 & 44.4 & 66.7 & 44.4 \\
\hline & Nonmusician gains & 38.1 & 28.6 & 28.6 & 38.1 & 28.6 & 23.8 & 33.3 & 38.1 \\
\hline & Nonmusician losses & 55.6 & 66.7 & 50.0 & 61.1 & 33.3 & 27.8 & 44.4 & 55.6 \\
\hline
\end{tabular}




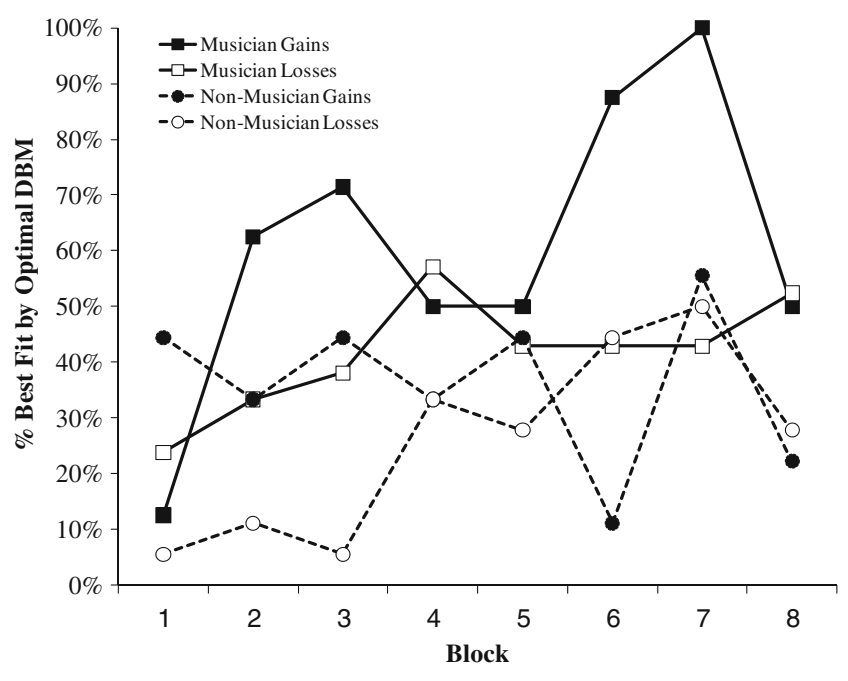

Fig. 3 Percentages of participants best fit by the optimal disjunctive rule in Experiment 1 in each block for self-identified musicians (solid lines) and nonmusicians (dotted lines), given the gains reward structure (filled markers) or the losses reward structure (open markers)

Overall, the pattern of changes across blocks in the percentages of participants best fit by the optimal disjunctive model paralleled the pattern of change in accuracy across blocks shown in Fig. 2. Notably, musicians given the gains reward structure showed a larger increase over blocks in the use of the optimal disjunctive rule than did musicians given the losses reward structure. This was not evident in a difference in the first block that the optimal disjunctive model fit the musicians' data best $(p>.05)$, but musicians with a gains reward structure were best fit by the optimal disjunctive model in more blocks $(M=4.75$ blocks $)$ than were musicians with a losses reward structure $(M=2.67$ blocks), $t(15)=1.96, p<.05$, one-tailed. Less consistent differences between gains and losses were observed for nonmusicians, for whom the numbers of blocks best fit by the optimal disjunctive model did not differ, $t(37)=1.7, p>$ .05 .

With respect to the overall best-fitting model (i.e., for choice data collapsed across blocks), the disjunctive models (optimal and suboptimal) were found to fit best for 67.9\% of participants, the unidimensional models (duration and frequency) fit best for only $7.1 \%$ of participants, and the random-responder model fit best for $25 \%$ of participants, $\chi^{2}(1)=7.14, p<.01$. This shows, more broadly, that although the task was difficult (i.e., $25 \%$ of the participants were best fit by the random-responder model), more participants were best fit by a disjunctive rule model than by any other rule. When self-identified musicians and nonmusicians were considered separately, $76.5 \%$ of the musicians were found to be best fit by the optimal model, while only $46.2 \%$ of the nonmusicians were best fit by the optimal model, $\chi^{2}(1)=4.40, p<.05$, thus showing an overall musicality effect. When musicians were further separated according to gains and losses, $100 \%$ of the musicians with the gains reward structure were found to be best fit by the optimal disjunctive model, while only $55 \%$ of the musicians with the losses reward structure were best fit by the optimal disjunctive model, $\chi^{2}(1)=4.65, p<.05$. Recall, as explained earlier, that the stochastic nature of the optimal model does not imply that a participant would have perfect accuracy, only that he or she would obtain the highest level of accuracy given the level of noise in their decision process. In contrast, no effect of reward structure was found on the percentages of nonmusicians best fit by the optimal disjunctive model (nonmusician gains, $42.9 \%$; nonmusician losses, $50 \%), \chi^{2}(1)=0.2$, n.s.

Relationship between self-report measures and perceptual classification performance

Finally, Table 5 summarizes the relationship between the pretest and posttest self-report measures and perceptual classification performance for the musician and nonmusician samples. There were no reliable correlations between responses to pretest questions and tone classification performance for musicians, but the pretest motivation rating was moderately correlated with performance for nonmusicians $(r=.36, p<.05)$. As expected, posttest measures of level of effort, attention, task difficulty, and task understanding tended to be correlated with performance, but not to the same degree for musicians and nonmusicians (see Table 5). With respect to the RFQ, PSWQ, BAI, and PANAS measures, there were two reliable relationships with tone classification performance: Better classification performance was associated with higher posttest positive affect (PA) scores $(r=.49, p<.01)$ and lower posttest negative affect (NA) scores $(r=-.33, p<.05)$.

\section{Discussion}

Self-identified musicians and nonmusicians completed a single-tone category-learning task that they were told was a test that musicians typically do better on than nonmusicians. Optimal classification performance required participants to learn an exclusive disjunctive classification rule, such that tones that were high in pitch and short in duration or low in pitch and long in duration were in one category, while tones that were low in pitch and short in duration or high in pitch and long in duration were in the other category. Musicians and nonmusicians were affected by the reward structure manipulation differently. Consistent with the hypothesis that musicians primed with a positive stereotype would adopt a promotion focus, musicians in the gains condition achieved higher accuracy levels and discovered the optimal disjunctive rule more readily than 
Table 5 Pearson correlations between responses to preand posttest items and overall proportions of correct tone classifications for musicians and nonmusicians
Significant differences: ${ }^{*} p<.05$, ${ }^{* *} p<.01$.

\begin{tabular}{lll}
\hline Pretest Items & Musicians & Nonmusicians \\
\hline How well do you think you will perform on this test? & .03 & .10 \\
How well do you think you will like the test? & .18 & .27 \\
How motivated are you to do well on the test? & -.40 & $.36^{*}$ \\
Posttest Items & & .26 \\
I am good at music. & -.12 & .12 \\
It is important for me to be good at music. & -.30 & -.02 \\
My musical ability is important to my identity. & -.27 & $.46^{* *}$ \\
How well did you perform overall? & $.92^{* *}$ & $.40^{*}$ \\
How well did you perform compared to musicians? & $.52^{*}$ & $.47^{* *}$ \\
How will did you perform compared to nonmusicians? & .38 & $.58^{* *}$ \\
Natural musical ability & -.06 & $.48^{* *}$ \\
Level of effort & -.03 & $.44^{* *}$ \\
Level of attention & .15 & -.23 \\
Level of task difficulty & $-.89^{* *}$ & $.54^{* *}$ \\
Level of task understanding & .41 & \\
\hline
\end{tabular}

did musicians in the losses condition. Conversely, nonmusicians showed minimal effects of the reward structure manipulation on performance. Comparing musicians and nonmusicians revealed that the musicians showed a performance advantage over nonmusicians for the gains reward structure, but not for the losses reward structure.

To provide a more direct test of the regulatory-fit hypothesis in the domain of auditory category learning, a second experiment was conducted in which we used the same task and reward structure manipulation, but directly primed regulatory focus. A promotion focus was primed by telling participants that good performance on the task would gain them entry into a raffle, while a prevention focus was primed by requiring participants to avoid poor performance on the task in order to maintain raffle entry. A regulatory-fit hypothesis predicted that promotion-primed participants would perform better with a gains reward structure than with a losses reward structure, while prevention-primed participants would perform better with a losses reward structure than with a gains reward structure.

\section{Experiment 2}

\section{Method}

\section{Participants and design}

A group of 58 undergraduate students with self-reported normal hearing from a large Midwestern university community participated in return for course credit in an undergraduate psychology course. The experiment had a 2 (regulatory focus: promotion vs. prevention) $\times 2$ (reward structure: gains vs. losses) $\times 8$ (block) mixed factorial design. Regulatory focus and reward structure were between-subjects factors, while block was a withinsubjects factor. Participants were randomly assigned to either the gains condition, in which they gained points for correct responses $(n=27)$, or a losses condition, in which they lost points for incorrect answers $(n=31)$, and were primed with either a promotion focus $(n=29)$ or a prevention focus $(n=29)$, yielding four between-subjects conditions (promotion-gains, $n=13$; promotion-losses, $n=$ 16; prevention-gains, $n=14$; prevention-losses, $n=15$ ).

\section{Stimuli and equipment}

The stimuli and equipment were identical to those of Experiment 1.

\section{Procedure}

The primary change in Experiment 2 was that, rather than telling participants that musicians typically outperformed nonmusicians on the task and then having them identify themselves as either a musician or a nonmusician, we randomly assigned participants to one of two regulatory focus conditions. Participants given the promotion focus prime were told that they would earn a raffle ticket with a 1 -in-20 chance of winning a $\$ 50$ cash prize if they performed well enough on the final block of the task. Participants given the prevention focus prime received a raffle ticket with a 1 -in-20 chance of winning a $\$ 50$ cash prize at the start of the experiment and were told that they would lose their raffle ticket if they failed to perform well enough in the final block of the task.

Participants then completed eight blocks of the tone classification task used in Experiment 1. After each block 
of 36 trials, participants were given feedback about their performance. If enough points were achieved in the gains condition to meet or exceed the criterion, or if participants successfully avoided the criterion in the losses condition, promotion-focused participants were told that they would have received a raffle ticket if this had been the final block of the experiment, and prevention-focused participants were told that they would have successfully avoided losing their raffle ticket if this had been the final block of the experiment. On the other hand, if not enough points were achieved in the gains condition or too many points were lost in the losses condition, promotion-focused participants were told that they would not have received a raffle ticket, and prevention-focused participants were told that they would have lost their raffle ticket if this had been the final block of the experiment. When the final block was completed, participants earned or kept (vs. did not earn or failed to keep) their raffle ticket, depending on their regulatory focus condition and performance.

After completing the perceptual classification task, participants completed a posttest questionnaire that assessed their ratings of natural musical ability $(1=$ very poor, $6=$ very good $)$, level of effort $(1=$ I did not try at all, $6=$ I tried my best $)$, level of attention $(1=I$ did not pay attention, $6=$ I paid full attention), level of task difficulty $(1=$ not difficult at all, $6=$ very difficult), and level of task understanding $(1=I$ did not understand at all, $6=I$ understood exactly what to do). The experiment lasted approximately $90 \mathrm{~min}$.

\section{Results}

\section{Perceptual classification performance}

Accuracy analyses Figure 4 shows proportions of correct tone classifications (PCs) for each of the eight blocks of trials for promotion-focus-primed participants (solid lines) and prevention-focus-primed participants (dotted lines) in the gains condition (solid markers) and the losses condition (open markers). A 2 (regulatory focus) $\times 2$ (reward structure) $\times 8$ (block) mixed-measures ANOVA on PCs revealed main effects of block, $F(7,378)=12.85, p<.01$, and regulatory focus, $F(1,54)=5.79, p<.01$, and a significant interaction between regulatory focus and reward structure, $F(1,54)=6.46, p=.01$. There was no main effect of reward structure, $F(1,54)=2.66, p=.1$, and no other significant interactions (all $p \mathrm{~s}>.17$ ). ${ }^{1}$ Accuracy was higher for promotion-focused $(M=.69, S D=.13)$ than for

\footnotetext{
${ }^{1}$ An ANOVA on $d^{\prime}$ in Experiment 2 similarly revealed main effects of block, $F(7,378)=6.28, p<.01$, and regulatory focus, $F(1,54)=4.8$, $p<.05$, and a significant interaction between regulatory focus and reward structure, $F(1,54)=6.6, p<.01$.
}

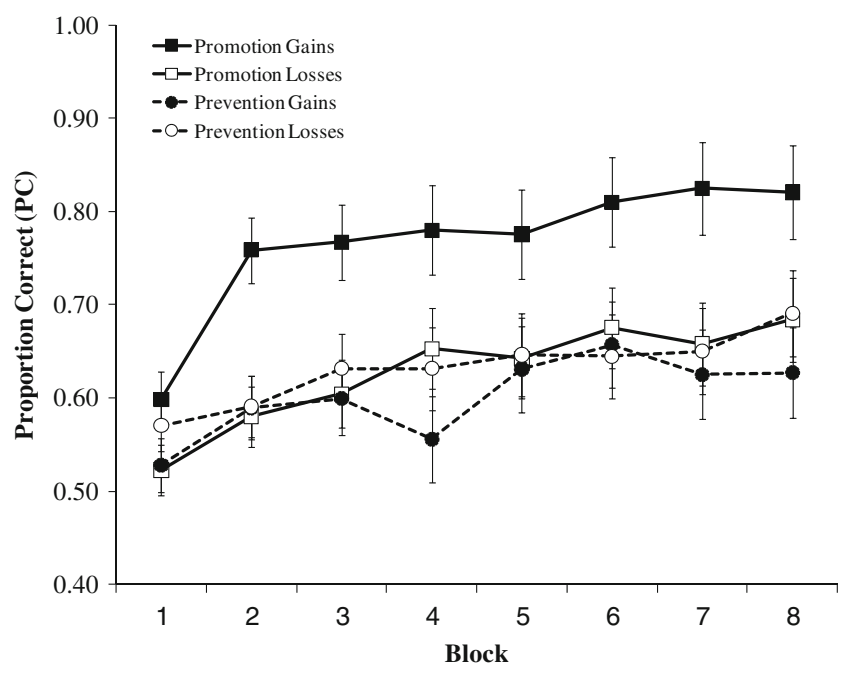

Fig. 4 Proportions of correct classification responses (PC) in Experiment 2 in each block for promotion-focus-primed participants (solid lines) and prevention-focus-primed participants (dotted lines), given the gains reward structure (filled markers) or the losses reward structure (open markers)

prevention-focused $(M=.62, S D=.13)$ participants, but differences in accuracy due to regulatory focus were qualified by a two-way interaction between regulatory focus and reward structure. Consistent with the regulatory-fit hypothesis, accuracy for promotion-focused participants (similar to self-identified musicians in Exp. 1) was higher in the gains condition $(M=.77, S D=.13)$ than in the losses condition $(M=.63, S D=.11), t(27)=3.20, p<.01$. Conversely, prevention-focused participants showed slightly higher accuracy in the losses condition $(M=.63, S D=.15)$ than in the gains condition $(M=.60, S D=.12)$, but the difference was not significant, $t(27)=-0.60, p=.55$.

Model-based analyses As in Experiment 1, three classes of DBMs were examined: unidimensional frequency and duration models, optimal and suboptimal disjunctive models, and a random-responder model (see Table 3). The models fit the data well, explaining $85.1 \%$ of variance, which is similar to the proportion of variance accounted for in Experiment 1. Table 6 summarizes the percentages of participants in each block who were best fit by the optimal disjunctive model, the suboptimal disjunctive model, the unidimensional frequency model, the unidimensional duration model, and the random-responder model, while Fig. 5 shows just the percentages of participants who were best fit by the optimal DBM for each of the eight blocks and each of the four groups (promotion-gains, promotion-losses, prevention-gains, prevention-losses). As in Experiment 1, the block-by-block changes in the percentages of participants best fit by the optimal disjunctive model (Fig. 5) paralleled the block-by-block change in the accuracy pattern (Fig. 4). Moreover, promotion-primed participants 
Table 6 Percentages of promotion- and preventionprimed participants in the gains and losses incentive conditions in Experiment 2 for which an optimal, a suboptimal, a unidimensional (UD) duration, a unidimensional frequency, or a random-responder model best fit the accuracy (as percentages correct) data according to the BIC metric

\begin{tabular}{llllllllll}
\hline & \multicolumn{2}{c}{ Block } & & & & & & & \\
\hline Model & Condition & 1 & 2 & 3 & 4 & 5 & 6 & 7 & 8 \\
Optimal & Promotion gains & 30.8 & 61.5 & 76.9 & 76.9 & 69.2 & 76.9 & 61.5 & 84.6 \\
& Promotion losses & 12.5 & 18.8 & 18.8 & 50.0 & 50.0 & 50.0 & 56.3 & 43.8 \\
& Prevention gains & 7.1 & 28.6 & 28.6 & 28.6 & 57.1 & 64.3 & 35.7 & 35.7 \\
& Prevention losses & 13.3 & 33.3 & 33.3 & 46.7 & 46.7 & 40.0 & 40.0 & 46.7 \\
Suboptimal & Promotion gains & 7.7 & 7.7 & 0.0 & 15.4 & 15.4 & 7.7 & 23.1 & 0.0 \\
& Promotion losses & 12.5 & 6.3 & 6.3 & 12.5 & 6.3 & 6.3 & 6.3 & 6.3 \\
& Prevention gains & 14.3 & 0.0 & 7.1 & 14.3 & 0.0 & 0.0 & 14.3 & 7.1 \\
& Prevention losses & 13.3 & 0.0 & 13.3 & 0.0 & 0.0 & 6.7 & 6.7 & 6.7 \\
UD Freq & Promotion gains & 15.4 & 7.7 & 7.7 & 7.7 & 7.7 & 7.7 & 7.7 & 7.7 \\
& Promotion losses & 31.3 & 31.3 & 31.3 & 12.5 & 18.8 & 18.8 & 18.8 & 31.3 \\
& Prevention gains & 28.6 & 42.9 & 35.7 & 28.6 & 14.3 & 0.0 & 28.6 & 14.3 \\
& Prevention losses & 53.3 & 26.7 & 26.7 & 40.0 & 20.0 & 40.0 & 26.7 & 26.7 \\
UD Dur & Promotion gains & 15.4 & 7.7 & 0.0 & 0.0 & 0.0 & 7.7 & 0.0 & 0.0 \\
& Promotion losses & 12.5 & 0.0 & 18.8 & 6.3 & 12.5 & 12.5 & 0.0 & 6.3 \\
& Prevention gains & 0.0 & 0.0 & 7.1 & 0.0 & 0.0 & 7.1 & 0.0 & 7.1 \\
& Prevention losses & 6.7 & 6.7 & 13.3 & 6.7 & 13.3 & 0.0 & 0.0 & 0.0 \\
& Promotion gains & 30.8 & 15.4 & 15.4 & 0.0 & 7.7 & 0.0 & 7.7 & 7.7 \\
& Promotion losses & 31.3 & 43.8 & 25.0 & 18.8 & 12.5 & 12.5 & 18.8 & 12.5 \\
& Prevention gains & 50.0 & 28.6 & 21.4 & 28.6 & 28.6 & 28.6 & 21.4 & 35.7 \\
& Prevention losses & 13.3 & 33.3 & 13.3 & 6.7 & 20.0 & 13.3 & 26.7 & 20.0 \\
\hline & & & & & & & & &
\end{tabular}

given a gains reward structure were best fit by the optimal disjunctive model in more blocks $[M=5.4$ vs. 3.0 blocks, $t$ $(27)=2.58, p<.05]$ and earlier $[M=2.6$ vs. 4.6 blocks, $t$ $(27)=-2.14, p<.05]$ than were promotion-primed participants given a losses reward structure. For preventionprimed participants, in contrast, reward structure did not appear to affect the number of blocks [prevention-gains, $M=$

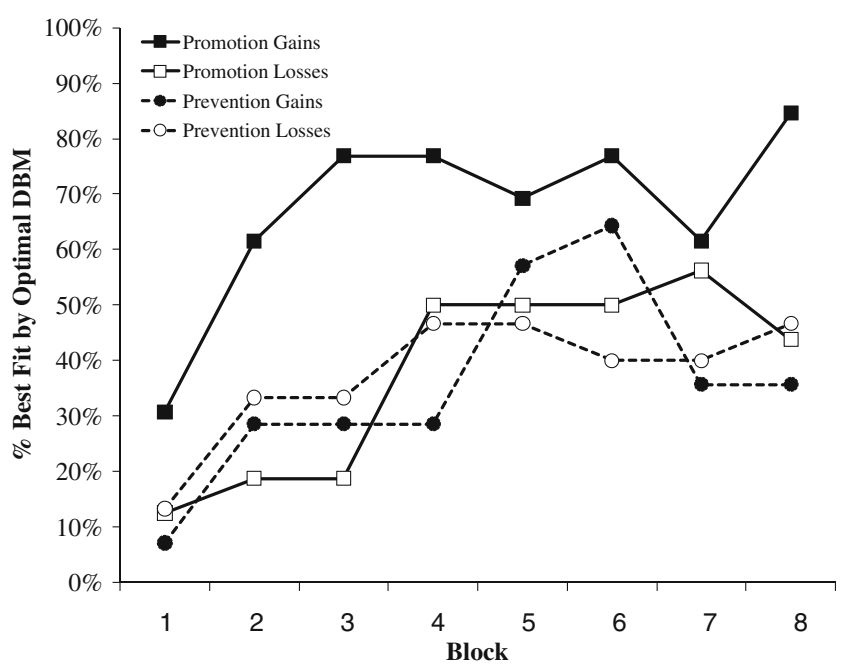

Fig. 5 Percentages of participants best fit by the optimal disjunctive rule in Experiment 2 in each block for promotion-focus-primed participants (solid lines) and prevention-focus-primed participants (dotted lines), given the gains reward structure (filled markers) or the losses reward structure (open markers)
2.86; prevention-losses, $M=3.0$ blocks; $t(27)=-0.15, p=$ .88] or how quickly participants learned the disjunctive rule [prevention-gains, $M=4.2$ blocks; prevention-losses, $M=$ 4.4 blocks; $t(27)=-0.18, p=.86]$.

With respect to the overall best-fitting model (i.e., for choice data collapsed across blocks), the disjunctive models (optimal and suboptimal) fit best for $58.6 \%$ of participants, the unidimensional models (duration and frequency) fit best for $25.9 \%$ of participants, and the random-responder model fit best for $15.5 \%$ of participants. As in Experiment 1, more than half of the participants were fit best by either the optimal or the suboptimal disjunctive model. When promotion-primed and prevention-primed participants were considered separately, $51.7 \%$ of the promotion-primed participants were found to be best fit by the optimal disjunctive model, whereas only $24.1 \%$ of the prevention-primed participants were best fit by the optimal disjunctive model, $\chi^{2}(1)=4.69, p<.05$. A further comparison of the gains versus losses conditions for the two regulatory focus conditions revealed a pattern that was consistent with a regulatory-fit hypothesis. For promotion-primed participants, $76.9 \%$ given a gains reward structure were best fit by the optimal disjunctive model, while only $31.3 \%$ given a losses reward structure were best fit by the optimal model, $\chi^{2}(1)=5.99, p<.025$. Conversely, for prevention-primed participants, $14.3 \%$ given a gains reward structure were best fit by the optimal disjunctive model, while $33.3 \%$ given a losses reward structure were best fit by the optimal model, $\chi^{2}(1)=1.45$, n.s. 


\section{Effects of regulatory focus and reward structure on posttest} item ratings

Finally, potential effects of regulatory focus and reward structure were assessed for items on the posttest questionnaire (see Table 7). With the exception of task understanding, there were no main effects of regulatory focus or reward structure, nor reliable interactions for any of the posttest items (all $p \mathrm{~s}>.1)$. For task understanding $(1=I$ did not understand at all, $6=$ I understood exactly what to do), promotion-primed participants gave higher task understanding ratings $(M=4.93, S D=1.39)$ than did preventionprimed participants $(M=3.76, S D=1.70), t(56)=4.91, p<$ .05 . Given that participants, by design, were not told the correct classification rule and had to learn it through trial and error, the effect of regulatory focus on task understanding was likely due to the better performance of promotionprimed participants.

\section{Discussion}

The results of the second experiment provide direct support for an effect of regulatory fit on auditory perceptual classification. For the promotion focus manipulation, participants were told that they would have an opportunity to earn entry into a $\$ 50$ raffle, while for the prevention focus manipulation participants were given a raffle ticket and needed to avoid poor performance to prevent loss of the ticket. Consistent with a regulatory-fit hypothesis, promotion-primed participants performed better in the gains condition (regulatory fit) than in the losses condition (regulatory nonfit). Prevention-primed participants tended to show the opposite pattern, but the differences observed between the gains and losses conditions with the prevention prime were not found to be reliable. Converging evidence was provided from the model-based analyses, which revealed a regulatory-fit advantage in participants' use of the optimal disjunctive rule.

\section{General discussion}

This article reported two experiments that used regulatory focus theory as a framework to begin to address whether musician advantages in assessments of auditory perception skills may partly reflect individual differences in motivational orientation. The general approach was based on Grimm et al. (2009), who in part were investigating whether performance on a visual classification task with an initially unknown conjunctive rule could be influenced by the regulatory fit between a primed stereotype (i.e., "men typically outperform women" or "women typically outperform men") and the reward structure of the task. Consistent with the view that participants given positive stereotypes would focus on goal attainment and adopt a promotion focus, while those given negative stereotypes would focus on avoiding poor performance and adopt a prevention focus, Grimm et al. found that women given positive stereotypes outperformed men given negative stereotypes when the women were experiencing a gains reward structure, but that the reverse effect was observed when the opposite stereotypes were primed.

The present study showed an analogous, but not identical, pattern of results when comparing musicians and nonmusicians who were primed with a positive and a negative stereotype, respectively, before completing a tone classification task with an initially unknown disjunctive rule. The primary hypothesis was that priming self-identified musicians with a positive stereotype (i.e., musicians typically outperform nonmusicians) would lead musicians to focus on goal attainment and adopt a promotion focus. Consistent with this hypothesis and similar to the pattern observed by Grimm et al. (2009), musicians outperformed nonmusicians in a gains reward structure (a regulatory fit), but not in a losses reward structure (regulatory nonfit). Model-based analyses further showed that musicians were quicker than nonmusicians to learn the optimal disjunctive rule, but only with a gains reward structure.

Table 7 Mean ratings $( \pm S D$ ) for self-report items for promotion-focus- and prevention-focus-primed participants in the gains and losses conditions

\begin{tabular}{lllll}
\hline Posttest Questionnaire & \multicolumn{2}{l}{ Promotion } & & \multicolumn{2}{c}{ Prevention } \\
\cline { 2 - 3 } & Gains & Losses & Gains & Losses \\
\hline Natural musical ability & $3.5(1.7)$ & $2.6(1.3)$ & $3.6(1.5)$ & $3.1(1.3)$ \\
Level of effort & $5.6(0.9)$ & $5.3(0.9)$ & $5.4(0.9)$ & $5.3(0.9)$ \\
Level of attention & $5.4(1.0)$ & $4.9(1.3)$ & $4.4(1.2)$ & $5.2(0.9)$ \\
Level of task difficulty & $4.2(1.4)$ & $4.0(1.4)$ & $3.6(1.6)$ & $3.9(1.2)$ \\
Level of task understanding & $4.8(1.5)$ & $5.0(1.3)$ & & 3.9 \\
\hline
\end{tabular}

Only task understanding differed between the conditions, with promotion-primed participants providing higher ratings than prevention-primed participants, $p<.05$. There were no effects of reward structure or interactions between reward structure and regulatory focus (all $p \mathrm{~s}>.1$ ). 
Nonmusicians, in contrast, did not appear to be affected by the prime. This might be explained by the fact that the effectiveness of priming a negative stereotype has been shown to depend on the importance that individuals place on the ability in question (e.g., women primed with a negative stereotype about math performance have been shown to be more affected by the prime if they assign high importance to mathematical ability; Cadinu et al., 2003). In line with this possibility, nonmusicians gave much lower ratings to the statements "It is important for me to be good at music" and "My musical ability is important to my identity" than did musicians.

Experiment 2 provided some direct support that regulatory fit impacts auditory perceptual classification and converging evidence that performance differences (or lack thereof) between musicians and nonmusicians in Experiment 1 were likely due to priming a difference in regulatory focus. Instead of telling participants that musicians typically outperformed nonmusicians on the task, participants were explicitly given promotion and prevention primes. Promotion-primed participants were told that they had an opportunity to be entered into a raffle with a 1-in-20 chance of winning if they performed well enough on the task, while prevention-primed participants were given a raffle ticket with a 1 -in-20 chance of winning and told that they would lose it if they failed to maintain a given level of performance. Consistent with a regulatory-fit hypothesis, promotion-primed participants showed greater accuracy levels and learned the disjunctive classification rule more quickly in the gains condition than in the losses condition, while prevention-primed participants showed only a slight performance advantage in the losses condition as compared to the gains condition.

The results from Experiment 2 are mostly consistent with the work of Maddox and colleagues, who have previously demonstrated interactions between regulatory focus and reward structure (i.e., regulatory fit) (Grimm et al., 2008; Maddox et al., 2006; Markman, Baldwin, \& Maddox, 2005; Markman, Maddox, \& Baldwin, 2007; Markman, Maddox, \& Worthy, 2006). Similar to the present investigation, Maddox et al. (2006) primed participants with a promotion or prevention focus and then had them complete a perceptual classification task (in this case, a visual rather than an auditory task) in which they either gained points for correct answers or lost points for incorrect answers. Critically, the perceptual classification tasks were chosen so that cognitive flexibility was either an advantage (Maddox et al., 2006, Exp.1) or a disadvantage (Maddox et al., 2006, Exps. 2 and 3). As in the present study, for a rule-based visual perceptual classification task in which cognitive flexibility was beneficial, individuals in a situationally induced regulatory fit learned the rule more quickly than (and outperformed) individuals in a regulatory nonfit.
One notable difference between the present results and the work of Maddox and colleagues is that although we found a regulatory-fit effect for the promotion-gains condition, a regulatory-fit effect was much less evident for the prevention-losses condition. The failure to find a robust prevention-losses fit effect for a rule-based auditory perceptual classification task is somewhat surprising, given that we used the same type of promotion and prevention primes and the same reward structure manipulation is in Maddox et al. (2006). There does not appear to be a straightforward explanation for this difference.

One factor that may have contributed to a weak prevention-losses fit effect in the present study is a chronic promotion focus bias that tends to be prevalent in collegestudent populations (Higgins, 2008). A participant bias toward a chronic promotion focus could have conceivably interacted with the situational regulatory focus primes, enhancing the effect of the promotion primes and weakening the efficacy of the prevention prime. Consistent with this possibility, we did observe a chronic promotion focus bias in Experiment 1, but there was little evidence that individual differences in chronic regulatory focus influenced performance or interacted with the reward structure manipulation. $^{2}$

A second factor that could be important to consider is task difficulty. Overall, auditory perceptual classification performance in the present study was relatively poorer than in some previous visual perceptual classification studies that have examined regulatory-fit effects (Maddox et al., 2006). In this regard, there is some evidence that participants may be particularly used to gains environments (see Grimm et al., 2009), which leads them to experience gains conditions as inherently easier than losses condition, with the fit (promotion-gains) group then experiencing a boost. If this were the case in the present study, one would expect this to be reflected in ratings of task difficulty or task

\footnotetext{
${ }^{2}$ Chronic regulatory focus was of particular interest in Experiment 1 because participants assigned themselves to either the musician or nonmusician samples, rather than being randomly assigned to promotion focus and prevention focus groups as in Experiment 2. For all participants, chronic promotion scores $(M=$ $19.4, S D=2.2$ ) were reliably higher than chronic prevention scores $(M=15.0, S D=2.8), t(55)=9.12, p<.01$, which is consistent with a promotion bias in the college student population. Musicians and nonmusicians, however, did not significantly differ in chronic promotion focus, $t(54)=-1.43, p=.16$, or chronic prevention focus, $t(54)=0.44, p=.66$. RFQ scores were also uncorrelated with classification accuracy (promotion score, $r=-.02, p=.88$; prevention score, $r=-.05, p=.69$ ). Finally, to assess whether chronic RFQ interacted with reward structure and could have had an impact on the pattern of results, the difference between the promotion and prevention scores on the RFQ was used to classify participants as having more of a chronic promotion or prevention focus. A 2 (chronic focus) $\times 2$ (reward structure) $\times 8$ (block) ANOVA yielded no main effect of chronic focus $(p=.78)$, nor any interactions with chronic focus (all $p \mathrm{~s}>.4$ ).
} 
understanding. However, reward structure (gains vs. losses) had no effect on either task difficulty or task understanding for both Experiments 1 and 2.

Another consideration that might help to explain a weak prevention-losses fit effect is the difference between the gains and losses reward structure manipulations. Specifically, in the gains reward structure, participants gained points for correct answers $(+2)$ but did not gain any points for incorrect answers $(+0)$. However, in the losses reward structure, participants lost points for correct $(-1)$ and for incorrect answers (-3). Pairing a prevention focus (which is characterized by a concern with maintenance and avoiding losses) with a reward structure that did not allow the participant to maintain the current performance level even when they were correct might have reduced a regulatory-fit effect with the prevention prime. Given that the losses reward structure was chosen to match Maddox et al. (2006), it does not explain the difference between the two studies, but nonetheless it is still possible that this particular choice of losses reward structure weakened a prevention-losses fit effect here. One approach to considering this possibility in future studies would be to titrate the numbers of points gained and lost for correct and incorrect responses.

A comparison of both experiments reveals strikingly similar patterns across studies, which serves to strengthen support for the conclusion that musicians in Experiment 1 adopted a promotion focus and experienced regulatory fit with the gains reward structure and nonfit with the losses reward structure. This conclusion has implications for distinguishing the effects of motivation from those of music ability at the two ends of the musical expertise spectrum. Impacts of music training on different aspects of perception and cognition have received increasing attention in the past decade or so (Cohen, 2000; Fujioka, Trainor, Ross, Kakigi, \& Pantev, 2004; Koelsch et al., 1999; Magne, Schön, \& Besson, 2006; Morrongiello \& Roes, 1990; Pechstedt, Kershner, \& Kinsbourne, 1989; Schön et al., 2004; Trainor, Shahin, \& Roberts, 2003). In this regard, it is notable that while many studies have reported robust and long-lasting perceptual benefits associated with music training, others have also failed to observe differences between musicians and nonmusicians (Bigand, 2003; Bigand \& Poulin-Charronnat, 2006; Grahn \& McAuley, 2009; Henry \& McAuley, 2009; Henry, McAuley, \& Zaleha, 2009). The fact that in the present study a relatively small change in the structure of the task (emphasizing gains vs. losses), combined with the simple prime "Musicians typically outperform nonmusicians" could have an impact on differences in task performance between musicians and nonmusicians highlights the need to understand better the interactions between motivational systems and basic cognitive processes in both the laboratory and more natural settings.
One implication of this study is the possibility that individual differences in motivation may also play an important role at the other end of the expertise spectrum. In particular, congenital amusia (or tone deafness) is a condition in which individuals have lifelong difficulty recognizing melodies without the aid of lyrics or detecting small pitch changes in music; this condition cannot be attributed to factors such as hearing loss, lack of exposure to music, or general intelligence (Ayotte, Peretz, \& Hyde, 2002). Some research has examined the influences of heredity (Peretz, Cummings and Dubé 2007) and brain structure (Hyde, Lerch, Zatorre, Griffiths, Evans, \& Peretz, 2007; Peretz, Brattico, Jarvenpaa, \& Tervaniemi, 2009; Peretz, Brattico, \& Tervaniemi, 2005) on amusia, but the role of individual differences in motivation orientation has been largely ignored.

As a step in this direction, we recently (McAuley, Henry, \& Tuft, 2011) examined the effects of regulatory fit on performance on the Montreal Battery of Evaluation of Amusia (MBEA; Peretz, Champod, \& Hyde, 2003). The MBEA was of interest because it is the primary assessment tool used to diagnose congenital amusia. To test a regulatory-fit hypothesis, we either gave musicians and nonmusicians instructional primes or explicitly primed a promotion or prevention focus. We then had participants complete a representative subtest of the MBEA that involved same-different judgments about melody pairs while either gaining points for correct answers (a gains condition) or losing points for incorrect answers (a losses condition). Consistent with a regulatory-fit hypothesis, promotion-primed participants achieved higher scores in a gains condition than in a losses condition, while prevention-primed participants performed better in a losses than in a gains condition.

Particularly relevant for the present investigation were two additional findings. Reward structure interacted with musicality, but in a manner opposite the one in the present study; musicians performed better when given a losses reward structure than when given a gains reward structure, thus appearing to adopt a prevention rather than a promotion focus when performing the task. Second, regulatory-fit effects with explicit promotion and prevention primes were generally stronger for musicians than for nonmusicians. The latter result notably converges with the overall more consistent regulatory-fit effects observed for musicians here, but the former result is at first glance puzzling.

Two methodological differences between McAuley et al. (2011) and the present study seem like good candidates to explain this difference. First, the instructional prime used with musicians and nonmusicians by McAuley et al. was that the "task was diagnostic of musical ability" rather than that "musicians typically outperform nonmusicians"; this might have particularly encouraged musicians to try to 
avoid doing poorly so that they would not be perceived as having low musical ability. Second, the task itself was one that was very familiar to musicians, and one in which they would thus have clear expectations about their performance. Notably, both methodological differences have been shown to be important for stereotype threat effects, which can be considered as part of a broader class of reputational threats. Our working hypothesis, in this regard, was that giving musicians a familiar task that they are told is diagnostic of musical ability represents a reputational threat and will encourage them to adopt a prevention, rather than a promotion, focus. Additional work is needed to test this possibility.

Another potentially fruitful line of work would be to contrast the effects of regulatory fit for individuals who score high and low on the MBEA. The key observation here is that there is a tendency for participants who do poorly on the MBEA to use a conservative response criterion (i.e., they tend to say that melodies are the same; Henry \& McAuley, 2011), which suggests that these participants may be adopting a prevention focus when performing the task. If this is the case, giving individuals who do poorly on the MBEA a losses reward structure would result in a state of regulatory fit and yield better performance than when these individuals are given a gains reward structure. This possibility is in line with recent work by Maddox, Filoteo, Glass, and Markman (2010), who considered how creating states of regulatory fit and nonfit might influence the proportions of individuals classified as "impaired" on the Wisconsin Card Sorting Task (WCST: Heaton, 1981). Consistent with a regulatory-fit hypothesis, individuals in a regulatory fit took fewer trials to adapt to a switched rule and made fewer perseverative responses than did individuals in a regulatory nonfit; moreover, fewer people in the regulatory-fit condition were classified as "impaired" on the WCST than in the regulatorynonfit condition.

Finally, although the focus of this study has been on musical expertise, it seems possible that the present findings could be more generally relevant for understanding effects of motivational orientation on task performance for other populations of experts (e.g., baggage screeners at the airport) placed in situations in which there is a desire either to show off their expertise (leading to a promotion focus orientation) or to avoid looking bad (leading to a prevention focus orientation). In both instances, a better understanding of how motivational orientation interacts with task characteristics can be used to frame tasks in a manner that will optimize task performance.

\section{Conclusions}

The present study represents a novel extension of regulatory focus theory to the auditory domain and provides evidence that the perceptual advantages typically associated with music training or musical ability have the potential to be reduced by considering the fit between an individual's regulatory focus and the reward structure of the task (i.e., whether or not participants gain points for correct responses or lose points for incorrect response). In two experiments, participants heard tones that varied in frequency and duration according to an initially unknown disjunctive rule and assigned tones to one of two categories, either gaining points for correct responses or losing points for incorrect responses. Experiment 1 revealed that selfidentified musicians learned the disjunctive rule more quickly than did nonmusicians in the gains condition, but that there was no musicality effect in the losses condition. Experiment 2 revealed a similar pattern of results for individuals primed with a promotion focus, as compared to those primed with a prevention focus. Overall, the findings are consistent with the hypothesis that musicians primed with a positive stereotype adopt a promotion focus when faced with a novel auditory perception assessment that affords musicians the opportunity to demonstrate their listening skills. Although musician versus nonmusician differences were observed with a gains reward structure, both the accuracy and model-based analyses revealed no musicality effects with a losses reward structure, supporting the more general view that effects of musicality (associated with either music ability or formal music training) are at least somewhat malleable, and likely partly depend on both motivational factors and task characteristics. Regulatory focus theory offers one approach that can be used to begin to disentangle the effects of individual differences in motivational orientation from those associated with music training or musical ability.

Author note Portions of this research were presented at the 10th Biennial Meeting of the Society for Music Perception and Cognition. The authors gratefully acknowledge Samantha Tuft and Bryan Grushcow for their contributions to this project.

\section{Appendix: Decision bound models}

In general, decision bound models (DBMs) explain how participants represent a stimulus in multidimensional space and ultimately make a categorization decision by assigning responses to regions of perceptual space (Ashby, 1992; Ashby \& Gott, 1988; Ashby \& Maddox, 1993; Maddox \& Ashby, 1993). The stimulus is defined by a set of coordinates in a physical multidimensional space with dimension $s$ (e.g., $s=2$ ). A vector $\mathbf{Y}_{i}$ represents the stimulus's coordinates in physical space. DBMs use a psychophysical function $\psi$ to map the physical space into 
the perceptual space. DBMs allow for a wide range of mappings to perceptual space, but in this set of experiments we have assumed a 1:1 mapping. DBMs allow for trial-bytrial (unbiased) variability in the percept so that

$\psi\left(\mathbf{Y}_{i}, \mathbf{e}_{p, i}\right)=\mathbf{X}_{p, i}=\mathbf{X}_{i}+\mathbf{e}_{p, i}$

where $\mathbf{e}_{p}$ is a random vector that represents sensory and perceptual noise. The vector $\mathbf{e}_{p}$ is assumed to be multivariate normal with covariance matrix $\sum_{p}$. In the models, the perceptual noise is stimulus invariant, so that the covariance matrix is the same for all stimuli. In the DBMs we used, we assumed zero covariance between dimensions, so that $\sum_{p} \sigma_{p}^{2} I$.

According to DBMs, participants divide up the psychophysical space with response criteria to make a decision. People may use an infinite number of possible decision rules. Table 3 describes the seven decision rules we used. A general description of each of the three classes of models (unidimensional, disjunctive, and random responder) is provided next.

\section{Unidimensional rule}

The unidimensional rule assumes that a respondent makes a categorization decision based on one dimension only. If a respondent only makes a decision on Dimension 1, then they set a criterion $\lambda_{1}$ on the perceived dimension. In general, with this model the probability of responding with Category $\mathrm{A}\left(R_{\mathrm{A}}\right)$, is

$\mathrm{P}\left(\mathrm{R}_{\mathrm{A}} \mid \mathbf{x}\right)=\mathrm{P}\left[\mathrm{x}_{1}+e_{\mathrm{p} 1}<\lambda_{1}+e_{\mathrm{c} 1}\right]$,

where $\lambda_{1}$ and $e_{\mathrm{c} 1}$ are the response bias and criterial error, and $e_{\mathrm{p} 1}$ is the perceptual noise on the first dimension. In the model, $e_{\mathrm{c} 1}$ and $e_{\mathrm{p} 1}$ are assumed to be independent and identically distributed. Equation A2 can be rewritten as

$P\left(R_{A} \mid \mathrm{X}\right)=\Phi\left(\frac{\lambda_{1}-x_{1}}{\sqrt{\sigma_{p}^{2}+\sigma_{c}^{2}}}\right)$

where $\Phi$ is the normal cumulative distribution function. The probability of responding $R_{\mathrm{B}}$ is $P\left(R_{\mathrm{B}} \mid \mathbf{x}\right)=1-P\left(R_{\mathrm{A}} \mid \mathbf{x}\right)$. In the models, we cannot identify $\sigma_{\mathrm{p}}$ and $\sigma_{\mathrm{c}}$ separately, so we fit only one noise parameter $\sigma^{2}=\sigma_{\mathrm{p}}{ }^{2}+\sigma_{\mathrm{c}}{ }^{2}$. Thus, each model has two free parameters: the variability parameter $\sigma$ and the decision bias parameter $\delta$.

For each dimension, we fit two unidimensional models. One version assumed that participants responded $\mathrm{A}$ if the perceived stimulus's first dimension value was less than the criterion, or otherwise they responded B (see Eq. A2). A second model assumed the opposite mapping. Unidimensional models based on the second dimension were developed in the same manner.
Disjunctive rule

The disjunctive rule was the optimal rule, in that the true categories in the experiment were defined with a disjunctive rule. Thus, participants who adopted a disjunctive rule would maximize accuracy. The decision rule required participants to determine whether the value on the first dimension $x_{1}$ was low or high and whether the value on the second dimension $x_{2}$ was low or high and then to combine those two separate dimensions to generate a response. Using the dimensions of duration and frequency, the optimal bounds required participants to use the following rule: "Respond ' $\mathrm{A}$ ' if the duration is short and the frequency is high or if the duration is long and the frequency is low; otherwise, respond 'B.' " As Maddox et al. (2006) pointed out, this rule requires an overt verbalizable rule that requires separate decisions on each decision, and thus should be more difficult for participants to implement in the nonfit condition due to their limited cognitive resources.

To calculate the probability of responding "A," $P\left(R_{\mathrm{A}} \mid \mathbf{x}\right)$, it is useful to divide the two-dimensional space into four quadrants using the two orthogonal decision criteria. Starting from the top right and moving counterclockwise, analogous to Cartesian quadrants, we label the quadrants I, II, III, and IV. The probability $P\left(R_{\mathrm{A}} \mid \mathbf{x}\right)$ is equal to the probability that stimulus $\mathbf{x}$ is perceived as falling into either of the two Cartesian quadrants II or IV,

$\mathrm{P}\left(\mathrm{R}_{\mathrm{A}} \mid \mathbf{x}\right)=\mathrm{P}(\mathrm{II} \mid \mathbf{x})+\mathrm{P}(\mathrm{IV} \mid \mathbf{x})$.

Analogously, the probability of responding " $\mathrm{B}$ " is

$\mathrm{P}\left(\mathrm{R}_{\mathrm{B}} \mid \mathbf{x}\right)=\mathrm{P}(\mathrm{I} \mid \mathbf{x})+\mathrm{P}(\mathrm{III} \mid \mathbf{x})$.

According to the general structure of DMBs, the probability of a stimulus falling into quadrant III (assuming criteria $\lambda_{1}$ and $\lambda_{2}$ on their respective dimensions) is

$\mathrm{P}(\mathrm{III} \mid \mathbf{x})=\mathrm{P}\left[x_{1}+e_{\mathrm{p} 1}<\lambda_{1}+e_{\mathrm{c} 1}, \mathrm{x}_{2}+e_{\mathrm{p} 2}<\lambda_{2}+e_{\mathrm{c} 2}\right]$.

Thus, because the dimensions are not correlated, this expression can be rewritten as

$P(\mathrm{III} \mid \mathrm{X})=\Phi\left(\frac{\lambda_{1}-x_{1}}{\sigma_{p}^{2}+\sigma_{c}^{2}}\right) \Phi\left(\frac{\lambda_{2}-x_{2}}{\sigma_{p}^{2}+\sigma_{c}^{2}}\right)$.

Using similar logic, the probability of the perceived stimulus falling into quadrant IV is

$P(\mathrm{IV} \mid \mathrm{X})=\Phi\left(\frac{x_{1}-\lambda_{2}}{\sqrt{\sigma_{p}^{2}+\sigma_{c}^{2}}}\right) \Phi\left(\frac{\lambda_{2}-x_{2}}{\sigma_{p}^{2}+\sigma_{c}^{2}}\right)$. 
The probability of the perceived stimulus falling into quadrant II is

$\mathrm{P}(\mathrm{II} \mid \mathrm{X})=\Phi\left(\frac{\lambda_{2}-x_{1}}{\sqrt{\sigma_{p}^{2}+\sigma_{c}^{2}}}\right) \Phi\left(\frac{x_{2}-\lambda_{2}}{\sqrt{\sigma_{p}^{2}+\sigma_{c}^{2}}}\right)$.

The probability of the perceived stimulus falling into quadrant $I$ is

$P(\mathrm{I} \mid \mathrm{X})=\Phi\left(\frac{x_{1}-\lambda_{2}}{\sqrt{\sigma_{p}^{2}+\sigma_{c}^{2}}}\right) \Phi\left(\frac{x_{2}-\lambda_{2}}{\sqrt{\sigma_{p}^{2}+\sigma_{c}^{2}}}\right)$.

Two disjunctive models were fit to the data. The optimal model set the criteria at $\lambda_{\mathrm{d}}=500 \mathrm{~ms}$ (duration) and $\lambda_{\mathrm{f}}=$ $466 \mathrm{~Hz}$ (frequency). These were the locations that were used to assign the category labels used in the experiment; thus, this model was optimal in that participants would maximize accuracy if they used it. Again, the perceptual and criterion noise parameters could not be separated, so one trial-by-trial parameter was fit. Thus, the optimal disjunctive model had one free parameter. The other model, the suboptimal disjunctive model, allowed the two criteria parameters to be free. After including the trial-to-trial variability parameter, this model had three free parameters.

\section{Random-response rule}

The random-response rule is essentially a statistical model in which the probability that a participant responded with Category A was set equal to the observed relative frequency of choosing the category. This model does not use the attributes of the stimulus, and thus over blocks the DBMs should do better than this model. This model had one free parameter, the observed relative frequency of responding "A."

Model fitting and comparison

In general, our goal was to use these DBMs as a rough model of the learning process (see also Maddox et al., 2006). To do so, we fit each DBM to each participant's data on a block-by-block basis and identified the best-fitting model for each block. If participants were learning the categories, then over the blocks we should see the DBM that assumes the correct decision rule (i.e., disjunctive) with the optimal criteria become the best-fitting model. All of the DBMs were fit to the responses using maximum likelihood methods. In the case of the four uniform DBMs and the two disjunctive DBMs, the best-fitting parameters were found with MATLAB's constrained nonlinear optimization routine based on a quasi-Newton approximation of the Hessian function. The parameter for the randomresponse rule was estimated directly from the data.
We used the Bayesian information criterion (BIC; Kass \& Raftery, 1995; Raftery, 1995; Schwarz, 1978; Wasserman, 2000) to make our model comparisons. The BIC was calculated for each model according to the following expression:

$B I C=-2 M L_{i}+j_{i} \log (n)$,

where $M L_{i}$ is the maximum log-likelihood of model $i, j$ is the number of parameters in the model, and $n$ is the number of observations. The model with the smallest BIC was selected as the best-fitting model. The number of parameters in the expression serves as a handicap for model complexity, where models with more parameters tend to overfit the data and therefore the BIC is handicapped more. As a rule of thumb, based on the BIC's Bayesian roots, 2 or less is interpreted as weak evidence, a difference of $2-6$ as positive evidence, a difference of $6-10$ as strong evidence, and a difference greater than 10 as very strong evidence for the particular model (Raftery, 1995; Wagenmakers, 2007).

\section{References}

Ashby, F. G. (1992). Multidimensional models of categorization. In F. G. Ashby (Ed.), Multidimensional models of perception and cognition (pp. 449-483). Hillsdale, NJ: Erlbaum.

Ashby, F. G., Alfonso-Reese, L. A., Turken, A. U., \& Waldron, E. M. (1998). A neuropsychological theory of multiple systems in category learning. Psychological Review, 105, 442-481. doi:10.1037/0033-295X.105.3.442

Ashby, F. G., \& Gott, R. E. (1988). Decision rules in the perception and categorization of multidimensional stimuli. Journal of Experimental Psychology. Learning, Memory, and Cognition, 14, 33-53. doi:10.1037/0278-7393.14.1.33

Ashby, F. G., \& Maddox, W. T. (1993). Relations between prototype, exemplar, and decision bound models of categorization. Journal of Mathematical Psychology, 37, 372-400. doi:10.1006/ jmps.1993.1023

Ashby, F. G., \& Maddox, W. T. (2005). Human category learning. Annual Review of Psychology, 56, 149-178. doi:10.1146/ annurev.psych.56.091103.070217

Ayotte, J., Peretz, I., \& Hyde, K. (2002). Congenital amusia: A group study of adults afflicted with a music-specific disorder. Brain, 125, 238-251. doi:10.1093/brain/awf028

Beck, A. T., Epstein, N., Brown, G., \& Steer, R. A. (1988). An inventory for measuring clinical anxiety: Psychometric properties. Journal of Consulting and Clinical Psychology, 56, 893897.

Bigand, E. (2003). More about the musical expertise of musically untrained listeners. Annals of the New York Academy of Sciences, 999, 304-312.

Bigand, E., \& Poulin-Charronnat, B. (2006). Are we "experienced listeners"? A review of the musical capacities that do not depend on formal musical training. Cognition, 100, 100-130. doi:10.1016/j.cognition.2005.11.007

Boersma, P., \& Weenink, D. (2005). Praat: Doing phonetics by computer (Version 4.4.14) [Computer program].

Brodscholl, J. C., Kober, H., \& Higgins, E. T. (2007). Strategies of self-regulation in goal attainment versus goal maintenance. European Journal of Social Psychology, 37, 628-648. 
Cadinu, M., Maass, A., Frigerio, S., Impagliazzo, L., \& Latinotti, S. (2003). Stereotype threat: The effect of expectancy on performance. European Journal of Social Psychology, 33, 267-285. doi:10.1002/ejsp.145

Cesario, J., \& Higgins, E. T. (2008). Making message recipients "feel right. Psychological Science, 19, 415-420.

Cesario, J., Higgins, E. T., \& Scholer, A. A. (2008). Regulatory fit and persuasion: Basic principles and remaining questions. Social and Personality Psychology Compass, 2, 444-463.

Chen, J. L., Penhune, V. B., \& Zatorre, R. J. (2008). Moving on time: Brain network for auditory-motor synchronization is modulated by rhythm complexity and musical training. Journal of Cognitive Neuroscience, 20, 226-239.

Cohen, A. J. (2000). Development of tonality induction: Plasticity, exposure, and training. Music Perception, 17, 437-459.

Erickson, M. A., \& Kruschke, J. K. (1998). Rules and exemplars in category learning. Journal of Experimental Psychology. General, 127, 107-140. doi:10.1037/0096-3445.127.2.107

Forster, J., Grant, H., Idson, L. C., \& Higgins, E. T. (2001). Success/ failure feedback, expectancies, and approach/avoidancemotivation: How regulatory focus moderates classic relations. Journal of Experimental Social Psychology, 37, 253-260.

Freitas, A. L., \& Higgins, E. T. (2002). Enjoying goal-directed action: The role of regulatory fit. Psychological Science, 13, 1-6. doi:10.1111/1467-9280.00401

Fujioka, T., Trainor, L. J., Ross, B., Kakigi, R., \& Pantev, C. (2004). Musical training enhances automatic encoding of melodic contour and interval structure. Journal of Cognitive Neuroscience, 16, 1010-1021. doi:10.1162/0898929041502706

Gaser, C., \& Schlaug, G. (2003). Brain structures differ between musicians and non-musicians. Journal of Neuroscience, 23, 9240-9245.

Grahn, J. A., \& McAuley, J. D. (2009). Neural bases of individual differences in beat perception. NeuroImage, 47, 1894-1903. doi:10.1016/j.neuroimage.2009.04.039

Grahn, J. A., \& Rowe, J. B. (2009). Feeling the beat: Premotor and striatal interactions in musicians and nonmusicians during beat perception. Journal of Neuroscience, 29, 7540-7548. doi:10.1523/JNEUROSCI.2018-08.2009

Grimm, L. R., Markman, A. B., Maddox, T. W., \& Baldwin, G. C. (2008). Differential effects of regulatory fit on category learning. Journal of Experimental Social Psychology, 44, 920-927.

Grimm, L. R., Markman, A. B., Maddox, W. T., \& Baldwin, G. C. (2009). Stereotype threat reinterpreted as a regulatory mismatch. Journal of Personality and Social Psychology, 96, 288-304.

Heaton, R. (1981). A manual for the Wisconsin Card Sorting Test. Odessa, FL: Psychological Assessment Resources.

Henry, M. J., \& McAuley, J. D. (2009). Evaluation of an imputed pitch velocity model of the auditory kappa effect. Journal of Experimental Psychology. Human Perception and Performance, 35, 551-564.

Henry, M. J., \& McAuley, J.D. (2011). A signal detection approach to the Montreal Battery of Evaluation of Amusia (MBEA). Manuscript under review

Henry, M. J., McAuley, J. D., \& Zaleha, M. F. (2009). Evaluation of an imputed pitch velocity model of the auditory tau effect. Perception \& Psychophysics, 71, 1399-1413.

Higgins, E. T. (1997). Beyond pleasure and pain. American Psychologist, 52, 1280-1300.

Higgins, E. T. (2000). Making a good decision: Value from fit. American Psychologist, 55, 1217-1230. doi:10.1037/0003066X.55.11.1217

Higgins, E. T. (2006). How regulatory fit creates value. In Cremer Dd, M. Zeelenberg, \& J. K. Murninghan (Eds.), Social psychology and economics (pp. 79-94). New York: Guilford Press.
Higgins, E. T. (2008). Culture and personality: Variability across universal motives as the missing link. Social and Personality Psychology Compass, 2, 608-634.

Higgins, E. T., Friedman, R. S., Harlow, R. E., Idson, L. C., Auduk, O. N., \& Taylor, A. (2001). Achievement orientations from subjective histories of success: promotion pride versus prevention pride. European Journal of Social Psychology, 31, 3-23.

Higgins, E. T., Idson, L. C., Freitas, A. L., Spiegel, S., \& Molden, D. C. (2003). Transfer of value from fit. Journal of Personality and Social Psychology, 84, 1140-1153. doi:10.1037/00223514.84.6.1140

Hyde, K., Lerch, J., Zatorre, R., Griffiths, T., Evans, A., \& Peretz, I. (2007). Cortical thickness in congenital amusia: When less is better than more. Journal of Neuroscience, 27, 13028.

Hyde, K. L., Lerch, J., Norton, A., Forgeard, M., Winner, E., Evans, A. C., et al. (2009). Musical training shapes structural brain development. Journal of Neuroscience, 29, 3019-3025. doi:10.1523/JNEUROSCI.5118-08.2009

Jäncke, L., Shah, N. J., \& Peters, M. (2000). Cortical activations in primary and secondary motor areas for complex bimanual movements in professional pianists. Cognitive Brain Research, 10, 177-183.

Jones, M. R., \& Yee, W. (1997). Sensitivity to time change: The role of context and skill. Journal of Experimental Psychology. Human Perception and Performance, 23, 693-709.

Kass, R. E., \& Raftery, A. E. (1995). Bayes factors. Journal of the American Statistical Association, 90, 773-795.

Kéri, S. (2003). The cognitive neuroscience of category learning. Brain Research Reviews, 43, 85-109. doi:10.1016/S0165-0173 (03)00204-2

Koelsch, S., Schröger, E., \& Tervaniemi, M. (1999). Superior preattentive auditory processing in musicians. NeuroReport, 10, $1309-1313$.

Latimer, A. E., Rivers, S. E., Rench, T. A., Katulak, N. A., Hicks, A., Hodorowski, J. K., et al. (2008). A field experiment testing the utility of regulatory fit messages for promoting physical activity. Journal of Experimental Social Psychology, 44, 826-832. doi:10.1016/j.jesp.2007.07.013

Maddox, W. T., \& Ashby, F. G. (1993). Comparing decision bound and exemplar models of categorization. Perception \& Psychophysics, 53, 49-49.

Maddox, W. T., Baldwin, G. C., \& Markman, A. B. (2006). A test of the regulatory fit hypothesis in perceptual classification learning. Memory \& Cognition, 34, 1377-1397. doi:10.3758/BF03195904

Maddox, W. T., Filoteo, J. V., Glass, B. D., \& Markman, A. B. (2010). Regulatory match effects on a modified Wisconsin Card Sort Task. Journal of the International Neuropsychological Society, 16, 352-359.

Maddox, W. T., \& Markman, A. B. (2010). The motivation-cognition interface in learning and decision making. Current Directions in Psychological Science, 19, 106-110.

Magne, C., Schön, D., \& Besson, M. (2006). Musician children detect pitch violations in both music and language better than nonmusician children: Behavioral and electrophysiological approaches. Journal of Cognitive Neuroscience, 18, 199-211.

Markman, A. B., Baldwin, G. C., \& Maddox, W. T. (2005). The interaction of payoff structure and regulatory focus in classification. Psychological Science, 16, 852-855. doi:10.1111/j.14679280.2005.01625.x

Markman, A. B., Maddox, W. T., Worthy, D. A., \& Baldwin, G. C. (2007). Using regulatory focus to explore implicit and explicit processing in concept learning. Journal of Consciousness Studies, 14, 132-155.

Markman, A. B., Maddox, W. T., \& Worthy, D. A. (2006). Choking and excelling under pressure. Psychological Science, 17, 944948. doi:10.1111/j.1467-9280.2006.01809.x 
McAuley, J. D., Henry, M. J., \& Tuft, S. (2011). Musician advantages in music perception: An issue of motivation, not just ability. Music Perception, 28, 505-518. doi:10.1525/ mp.2011.28.5.505

McAuley, J. D., \& Semple, P. (1999). The effect of tempo and musical experience on perceived beat. Australian Journal of Psychology, $51,176-187$.

Meyer, T. J., Miller, M. L., Metzger, R. L., \& Borkovec, T. D. (1990). Development and validation of the Penn State worry questionnaire. Behaviour Research and Therapy, 28, 487-495.

Morrongiello, B. A., \& Roes, C. L. (1990). Developmental changes in children's perception of musical sequences: Effects of musical training. Developmental Psychology, 26, 814-820. doi:10.1037/ 0012-1649.26.5.814

Musacchia, G., Sams, M., Skoe, E., \& Kraus, N. (2007). Musicians have enhanced subcortical auditory and audiovisual processing of speech and music. Proceedings of the National Academy of Sciences, 104, 15894-15898.

Pechstedt, P. H., Kershner, J., \& Kinsbourne, M. (1989). Musical training improves processing of tonality in the left hemisphere. Music Perception, 6, 275-298.

Peretz, I., Brattico, E., Jarvenpaa, M., \& Tervaniemi, M. (2009). The amusic brain: In tune, out of key, and unaware. Brain, 132, $1277-1286$.

Peretz, I., Brattico, E., \& Tervaniemi, M. (2005). Abnormal electrical brain responses to pitch in congenital amusia. Annals of Neurology, 58, 478-482.

Peretz, I., Champod, A. S., \& Hyde, K. L. (2003). Varieties of musical disorders: The Montreal Battery of Evaluation of Amusia. Annals of the New York Academy of Sciences, 999, 58-75.

Peretz, I., Cummings, S., \& Dubé, M. (2007). The genetics of congenital amusia (tone deafness): A family-aggregation study. American Journal of Human Genetics, 81, 582-588.

Raftery, A. E. (1995). Bayesian model selection in social research. Sociological Methodology, 25, 111-163.

Schlaug, G., Jäncke, L., Huang, Y., Staiger, J. F., \& Steinmetz, H. (1995). Increased corpus callosum size in musicians. Neuropsychologia, 33, 1047-1055. doi:10.1016/0028-3932(95)00045-5
Schlaug, G., Norton, A., Overy, K., \& Winner, E. (2005). Effects of music training on the child's brain and cognitive development. Annals of the New York Academy of Sciences, 1060, 219-230.

Schön, D., Magne, C., \& Besson, M. (2004). The music of speech: Music training facilitates pitch processing in both music and language. Psychophysiology, 41, 341-349.

Schwarz, G. (1978). Estimating the dimension of a model. The Annals of Statistics, 6, 461-464.

Spiegel, S., Grant-Pillow, H., \& Higgins, E. T. (2004). How regulatory fit enhances motivational strength during goal pursuit. European Journal of Social Psychology, 34, 39-54.

Strait, D. L., Kraus, N., Skoe, E., \& Ashley, R. (2009). Musical experience and neural efficiency-Effects of training on subcortical processing of vocal expressions of emotion. European Journal of Neuroscience, 29, 661-668. doi:10.1111/j.14609568.2009.06617.x

Trainor, L., Shahin, A., \& Roberts, L. (2003). Effects of musical training on the auditory cortex in children. Annals of the New York Academy of Sciences, 999, 506-513.

Wagenmakers, E.-J. (2007). A practical solution to the pervasive problems of $p$ values. Psychonomic Bulletin \& Review, 14, 779804. doi:10.3758/BF03194105

Wasserman, L. (2000). Bayesian model selection and model averaging. Journal of Mathematical Psychology, 44, 92-107.

Watson, D., Clark, L. A., \& Tellegen, A. (1988). Development and validation of brief measures of positive and negative affect: The PANAS scales. Journal of Personality and Social Psychology, 54, 1063-1070. doi:10.1037/0022-3514.54.6.1063

Werth, L., \& Foerster, J. (2007). How regulatory focus influences consumer behavior. European Journal of Social Psychology, 37, 33-51.

Wong, P. C. M., Skoe, E., Russo, N. M., Dees, T., \& Kraus, N. (2007). Musical experience shapes human brainstem encoding of linguistic pitch patterns. Nature Neuroscience, 10, 420-422.

Worthy, D. A., Markman, A. B., \& Maddox, W. T. (2009). What is pressure? Evidence for social pressure as a type of regulatory focus. Psychonomic Bulletin \& Review, 16, 344-349. doi:10.3758/PBR.16.2.344 